\title{
Barriers and facilitators to the uptake of new medicines into clinical practice: a systematic review
}

Kristina Medlinskiene ${ }^{1,2^{*}}$ (D) Justine Tomlinson ${ }^{1,2}$, luri Marques ${ }^{1}$, Sue Richardson ${ }^{3}$, Katherine Stirling ${ }^{2}$ and Duncan Petty ${ }^{1}$

\begin{abstract}
Background: Implementation and uptake of novel and cost-effective medicines can improve patient health outcomes and healthcare efficiency. However, the uptake of new medicines into practice faces a wide range of obstacles. Earlier reviews provided insights into determinants for new medicine uptake (such as medicine, prescriber, patient, organization, and external environment factors). However, the methodological approaches used had limitations (e.g., single author, narrative review, narrow search, no quality assessment of reviewed evidence). This systematic review aims to identify barriers and facilitators affecting the uptake of new medicines into clinical practice and identify areas for future research.

Method: A systematic search of literature was undertaken within seven databases: Medline, EMBASE, Web of Science, CINAHL, Cochrane Library, SCOPUS, and PsychINFO. Included in the review were qualitative, quantitative, and mixed-methods studies focused on adult participants (18 years and older) requiring or taking new medicine(s) for any condition, in the context of healthcare organizations and which identified factors affecting the uptake of new medicines. The methodological quality was assessed using QATSDD tool. A narrative synthesis of reported factors was conducted using framework analysis and a conceptual framework was utilised to group them.

Results: A total of 66 studies were included. Most studies $(n=62)$ were quantitative and used secondary data $(n=$ 46) from various databases, e.g., insurance databases. The identified factors had a varied impact on the uptake of the different studied new medicines. Differently from earlier reviews, patient factors (patient education, engagement with treatment, therapy preferences), cost of new medicine, reimbursement and formulary conditions, and guidelines were suggested to influence the uptake. Also, the review highlighted that health economics, wider organizational factors, and underlying behaviours of adopters were not or under explored.
\end{abstract}

\footnotetext{
*Correspondence: k.medlinskiene1@bradford.ac.uk;

kristina.medlinskiene@nhs.net

${ }^{1}$ Medicine Optimisation Research Group, School of Pharmacy and Medical Sciences, University of Bradford, Richmond Road, Bradford BD7 1DP, UK

${ }^{2}$ Medicine Management and Pharmacy Services, Leeds Teaching Hospitals NHS Trust, Leeds, UK

Full list of author information is available at the end of the article
}

(c) The Author(s). 2021 Open Access This article is licensed under a Creative Commons Attribution 4.0 International License, which permits use, sharing, adaptation, distribution and reproduction in any medium or format, as long as you give appropriate credit to the original author(s) and the source, provide a link to the Creative Commons licence, and indicate if changes were made. The images or other third party material in this article are included in the article's Creative Commons licence, unless indicated otherwise in a credit line to the material. If material is not included in the article's Creative Commons licence and your intended use is not permitted by statutory regulation or exceeds the permitted use, you will need to obtain permission directly from the copyright holder. To view a copy of this licence, visit http://creativecommons.org/licenses/by/4.0/ The Creative Commons Public Domain Dedication waiver (http://creativecommons.org/publicdomain/zero/1.0/) applies to the data made available in this article, unless otherwise stated in a credit line to the data. 
Conclusion: This systematic review has identified a broad range of factors affecting the uptake of new medicines within healthcare organizations, which were grouped into patient, prescriber, medicine, organizational, and external environment factors. This systematic review also identifies additional factors affecting new medicine use not reported in earlier reviews, which included patient influence and education level, cost of new medicines, formulary and reimbursement restrictions, and guidelines.

Registration: PROSPERO database (CRD42018108536).

Keywords: New medicines, Uptake, Implementation, Systematic review, Innovation implementation, Healthcare organizations

\section{Introduction}

The uptake of an evidence-based intervention in clinical practice can take on average 17 years before it becomes part of a routine practice [1]. In healthcare, medicines are deemed to be the most common therapeutic intervention requiring significant funds from the system [2]. The slow uptake of cost-effective and novel medicines can delay improvements in patient health outcomes, healthcare efficiency, and even lessen the international competitiveness of the country in the life sciences sector [2-4]. For instance, in the United Kingdom (UK), the relative uptake of nationally recommended new medicines often lags behind other comparative countries' health systems such as Australia, Canada or France [5].

There is a considerable amount of scientific literature exploring why the implementation of evidence-based interventions succeeds or fails within a complex healthcare environment [6]. Factors affecting implementation outcomes have been grouped into patient, provider, innovation, structural and organizational factors [7]. At the patient level, earlier reviews indicated patients' socio-demographic and economic characteristics influenced the uptake of new medicines [8-10]. However, patients' influence through their involvement in decisionmaking was relatively unexplored [8-10]. At provider level, prescribers' scientific orientation and prescribing habits were suggested to affect uptake [10]. Furthermore, innovation level factors, such as effectiveness, safetyprofile, convenience, and therapeutic novelty of new medicines were considered important aspects. Reviews concluded that cost was of low importance [8-10], but cost could be a factor in current healthcare systems as balancing increasing expenditure on medicines and available funding is becoming harder [2]. At an organizational level, mainly the impact of an organization's characteristics, e.g., size, ownership, was suggested to have limited impact $[8,10]$. Finally, structural level features, such as peer influence, pharmaceutical detailing, scientific literature and meetings, and regulatory pressures were identified as potential factors [8-10].
Although these earlier reviews provided some insight into the determinants of new medicine uptake, the methodological approaches had limitations (e.g., single author, narrative review, narrow search, no quality assessment of reviewed evidence). Also, healthcare systems have changed rapidly over the last ten years with increasing focus on patient-centred care and patient involvement in decision-making [11], use of medicines [2], expenditure on medicines [2], and new policies being developed to improve patient access to new medicines [12]. Studies in earlier reviews might not have captured all factors relevant to current healthcare systems and hence an updated review is warranted. This review, therefore, aims to identify barriers and facilitators affecting the uptake of new medicines into clinical practice, including areas for future research. Also, the review sought to provide more insight on the factors unexplored in earlier reviews such as patient influence and cost of new medicines.

\section{Methods \\ Protocol and registration}

A protocol for this review was registered on PROSPERO (Registration number: CRD42018108536) [13]. The conduct of the systematic review was guided by the Preferred Reporting Items for Systematic Review and MetaAnalysis (PRISMA) statement [14] (see Additional file 1 for the PRISMA checklist).

\section{Eligibility criteria}

The inclusion criteria were established using the PICOS framework [15]. Eligible studies focused on adult participants (18 years and older) requiring or taking any new medicine(s) for any condition in the context of healthcare organizations. The World Health Organization definition of health innovation was used to define 'new medicine' as new or improved pharmaceutical product which improved people's health and aimed to "add value in the form of improved efficiency, effectiveness, quality, sustainability, safety and/or affordability" [16]. Studied 
healthcare organizations were primary or secondary care. Eligible studies identified factors affecting (impeding or facilitating) the uptake of new medicines. The authors of this review considered uptake as the use of a new medicine within a healthcare organization within five years after it had been approved by the regulatory agency of the country where the study was conducted. Studies that only reported prescribing trends and/or patient demographics (age, gender) and clinical comorbidities were excluded. Qualitative, quantitative, or mixed-methods empirical studies published in English were eligible. Grey literature (conference proceedings, theses), review articles, clinical guidelines, and incomplete studies were excluded.

As healthcare systems have changed rapidly over the last ten years in relation to medicine use [2, 11, 12], studies from 2008 and onwards were included in the review to capture studies more relevant to current healthcare systems. Also, the review had a broad search strategy over seven databases, thus the time period limitation allowed to process a manageable number of studies yielded by the search.

\section{Information sources}

The search was conducted in seven electronic databases: Medline, EMBASE, Web of Science, CINAHL, Cochrane Library, SCOPUS, and PsychINFO. Hand-searching was conducted using Google Scholar, reference lists, and forward citations of included studies and relevant systematic reviews to identify relevant studies that were inaccurately indexed or unindexed.

\section{Search}

The search strategy was developed in collaboration with a subject librarian at the University of Bradford. The search was completed on 4 September 2018 and updated on 23 April 2020. The search terms were developed from four search categories: 'uptake', 'new medicine', 'healthcare organization', and 'barriers and facilitators' (see Additional file 2 for Medline search strategy).

\section{Study selection}

After the removal of duplicates using the reference management software (EndNote $\left.\mathrm{X} 7^{\circ}\right)$, one reviewer $(\mathrm{KM})$ independently screened titles and abstracts. The second reviewer (JT) screened rejected articles after titles and abstract screening to minimise the removal of potentially relevant studies [17]. Two reviewers (KM, JT) independently reviewed full-texts of potentially relevant studies. The first reviewer (KM) screened the reference lists and forward citations, and the second reviewer (JT) independently reviewed studies deemed to meet the eligibility criteria. Any disagreements were discussed to reach a consensus. If consensus was not reached, the third reviewer (IM) reviewed disagreements.

\section{Data collection process and data items}

A standardised proforma was developed by the research team and piloted with five studies before being finalised. One reviewer (KM) independently extracted data for $100 \%$ of the studies and the second reviewer (JT) independently checked the data extraction forms for accuracy and completeness. Any disagreements were discussed to reach a consensus. Abstracted data included citation information, study information (aim, design, data source, setting), studied new medicine, participant details, findings relevant to this review, funding source and reported conflict of interest. Lead authors of the studies were contacted via e-mail to provide missing or additional data if required.

\section{Risk of bias in individual studies}

Two independent reviewers (KM and JT or IM) assessed risk of bias of included individual studies using the Quality Assessment Tool for Studies with Diverse Designs (QATSDD) [18]. The QATSDD tool consists of 16 criteria and is validated to assess studies with heterogeneous study designs. The following aspects of studies were examined: theoretical framework; aims and objectives; research setting; sample size and representativeness; data collection procedure and rationale; recruitment; appropriateness, reliability and validity of data analysis tools or process; user involvement; strengths and limitations. Reviewers scored each study on a scale of 0 (not at all/not stated) to 3 (complete/explicitly stated) against each criterion. The maximum score was of 42 for quantitative and qualitative studies and 46 for mixed-methods studies. Disagreements were resolved through discussion or by a third reviewer (KM, JT, or IM). The total score for each study was calculated by adding scores for each criterion and expressed as a percentage $(0-100 \%)$. Studies with scores $<50 \%$ were classed as low, 50 to $>70 \%$ as moderate, and $>70 \%$ as high-quality studies. Although the low methodological quality studies were not excluded, they were given less weight in the synthesis of results and conclusions.

\section{Synthesis of results}

The meta-analysis was not feasible due to the heterogeneity of study designs and methods used. Therefore, a narrative synthesis using a 'best fit' framework synthesis [19] was conducted to summarise the findings of reviewed studies.

'Best fit' framework synthesis is based on framework analysis [20] and is thought to bring a more transparent and pragmatic process than other more interpretive forms of synthesis [19]. It is a two-stage synthesis 
process. Firstly, an initial framework of preliminary themes was identified against which the data from the reviewed studies would be mapped. The initial framework used in this review was a multi-level framework by Chaudoir et al., which was developed by collecting implementation success factors for health innovations from multiple previous frameworks [7]. The themes in the Chaudoir et al. [7] framework were patient, provider, innovation, structural, and organizational.

The second stage of the 'best fit' framework synthesis involved reviewing the studies meeting the inclusion criteria and coding the identified factors affecting the uptake of new medicines against the themes in the multi-level framework by Chaudoir et al. [7]. The reviewing and summarising of the coded data were completed using NVivo11 software. New themes were created for data that could not be coded against the framework through a process of interpretation similar to thematic analysis [19].

Two reviewers (KM, IM) independently coded the material, and any discrepancies were resolved through discussion. Identified factors were finalised in a team discussion (KM, SR, KS, DP).

\section{Results}

\section{Study selection}

The study selection process is summarised in the PRISMA flowchart (Fig. 1). Of 43,697 unique citations identified in the search strategy, and an additional 22 studies retrieved through alternative methods, 66 studies were eligible for inclusion.

\section{Study characteristics}

Study characteristic of included studies are presented in Table 1 . Most of the studies $(n=62)$ used quantitative methods $[21-49,51-65,68-79,81-86]$; three were qualitative $[50,67,80]$, and one was mixed-methods [66]. The predominant source of data collection was secondary data $(n=46)$ from various databases and registries $[22-25,27,29-34,36,40-44,46,48,51-64,66$, $70-72,74,78,79,81-85,87]$. Other studies $(n=17)$ used surveys [26, 44, 47, 67, 68, 73], interviews [21, 26, $37,50,57,66,67,76,80]$, patients' medical records [28, 45], prescriptions from community pharmacies [75], observations [35], or a focus group [80] to collect primary data. Two studies [38, 49] used both primary and

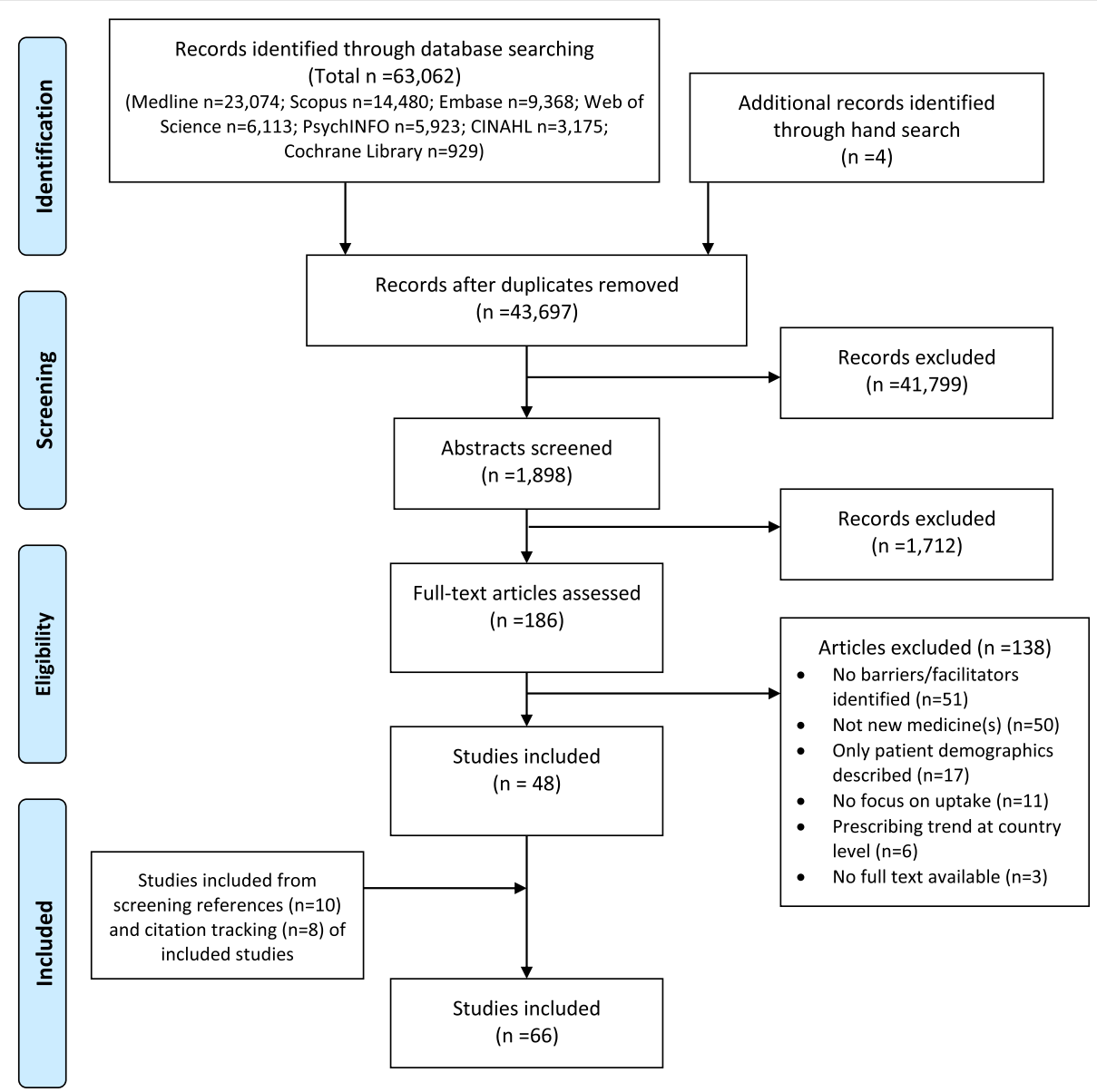

Fig. 1 PRISMA flow diagram showing the systematic literature search and screening process 
secondary data. Studied new medicines were from twenty different therapeutic classes and five studies described medicines as newly marketed.

\section{Risk of bias within studies}

The methodological quality of studies ranged from 45 to $81 \%$, with a mean score of $67 \%$ (see Additional file 3, Table S1). Two studies were deemed to be low, 38 medium, and 26 high quality. The most prominent methodological weaknesses were lack of reporting reliability and validity of data measurement tools used in quantitative studies and reliability of analytical process used in qualitative studies. There was no evidence of pilot testing or user involvement across all studies (Table 2).

\section{Synthesis of results}

Factors affecting the uptake of new medicines were grouped into five thematic areas: patient, prescriber, medicine, organizational and external environment factors. Summary of main thematic areas with subthemes are shown in Table 3.

The thematic area(s) in each included study is reported in Additional file 4, Table S2. External environment, organizational, patient and prescriber factors were reported most frequently ( $n=36, n=34, n=31$ and $n=$ 29 studies respectively) and medicine factors $(n=18)$ were the least. Summary of factors affecting the uptake of new medicines referred to in the reviewed studies is displayed in Table 4.

\section{Patient-level factors}

\section{Demographic characteristics $(n=21)$}

Studies reported mixed results of patients' age, gender, and ethnicity impact on the uptake of new medicines. Some prescribers tended to prescribe new medicines to younger $[22,28,35,51,52,58,63,71,72,74,78]$, male [22, 72, 74, 79], female [82], or white ethnicity [61] patients. Others observed use of new medicine in older patients $[25,32,70,82$, $85]$, mixed impact of ethnicity [25, 35, 51, 72, 74, 78], or suggested that patients' age $[60,73,79]$, gender $[28,29,58$, $60,71,73,78]$, ethnicity $[58,70,82]$ had no impact on prescribing decisions. Studies were medium to high quality and one study [79] was low quality.

\section{Socioeconomic status $(n=21)$}

Some prescribers reported that patients' socioeconomic factors [68], which included education, income, health insurance plan, residential area, influenced their prescribing decisions. Some findings suggested patients with a higher level of education were more likely to receive new medicines [32, 35, 43, 78], regardless of their age, gender, education, type of residential area, number of medicines used, and comorbidity [43]. However, one study [82] observed no impact of patient education level.
All studies were high-quality. Prescribers also considered affordability of new medicines by patients [50]. Some studies suggested patients with higher income or ability to pay out-of-pocket expenses were more likely to receive a new medicine [22, 32, 45, 47, 51, 71, 80, 82, 85], but one study observed no difference [25]. Only three studies were high-quality [32, 71, 82]. Furthermore, patients with private health insurance plans covering prescription medicines and medical care services were reported to have a greater access to new medicines [22, $25,35,45,58,62,72,74,78]$; two studies were highquality $[35,78]$. Lastly, some studies indicated that patients living in a capital city [43], urban [70], or more affluent areas [85] were more likely to receive new medicines; two studies were high-quality $[43,70]$.

\section{Health status $(n=21)$}

Prescribers highlighted that patients' clinical characteristics and comorbidities influenced new medicines use [50]. Some prescribers reported prescribing new medicines for patients with more severe disease $[34,35,46,63$, 82] or polypharmacy [22, 25, 43]; five studies were highquality $[34,35,43,46,82]$. Other low to high quality studies reported new medicines use in patients with fewer comorbidities, or less severe conditions $[22,28,51,72-74$, $78,79]$, and no polypharmacy or concomitant use of medicines increasing the risk of side effects $[28,72,73,79]$. Medium to high quality studies reported patient's poor response to the current treatment encouraged [47, 70, 80] and that patient's satisfaction with the existing treatment discouraged new medicine use [32].

\section{Patient engagement with treatment $(n=5)$}

Some prescribers stated that patients' requests for a new medicine $[47,61,80]$ and interest in it [67], adherence to current treatment $[50,80]$ and monitoring [47] were influential in prescribing decisions. Some prescribers were described as aiming for a shared decision-making thus patients' therapy preference and compatibility with their lifestyle [50] shaped prescribing decisions. Only one study was high-quality [61].

\section{Prescriber factors}

Socio-demographic characteristics $(n=11)$

Medium to high quality studies suggested younger [33, $48,53,62]$ or older [ 27,42$]$, male [23, $42,48,85]$, graduating from a top-20 medical $[23,62]$ or foreign medical school [48] prescribers were earlier adopters. Other medium to high quality studies reported that age [29, 42, $48]$, gender [29, 62], prescribers' length of practice [35, 85], graduating from a top- 20 medical school $[48,62]$ did not influence prescribing decisions. A mediumquality study indicated general practitioners' (GPs) who 


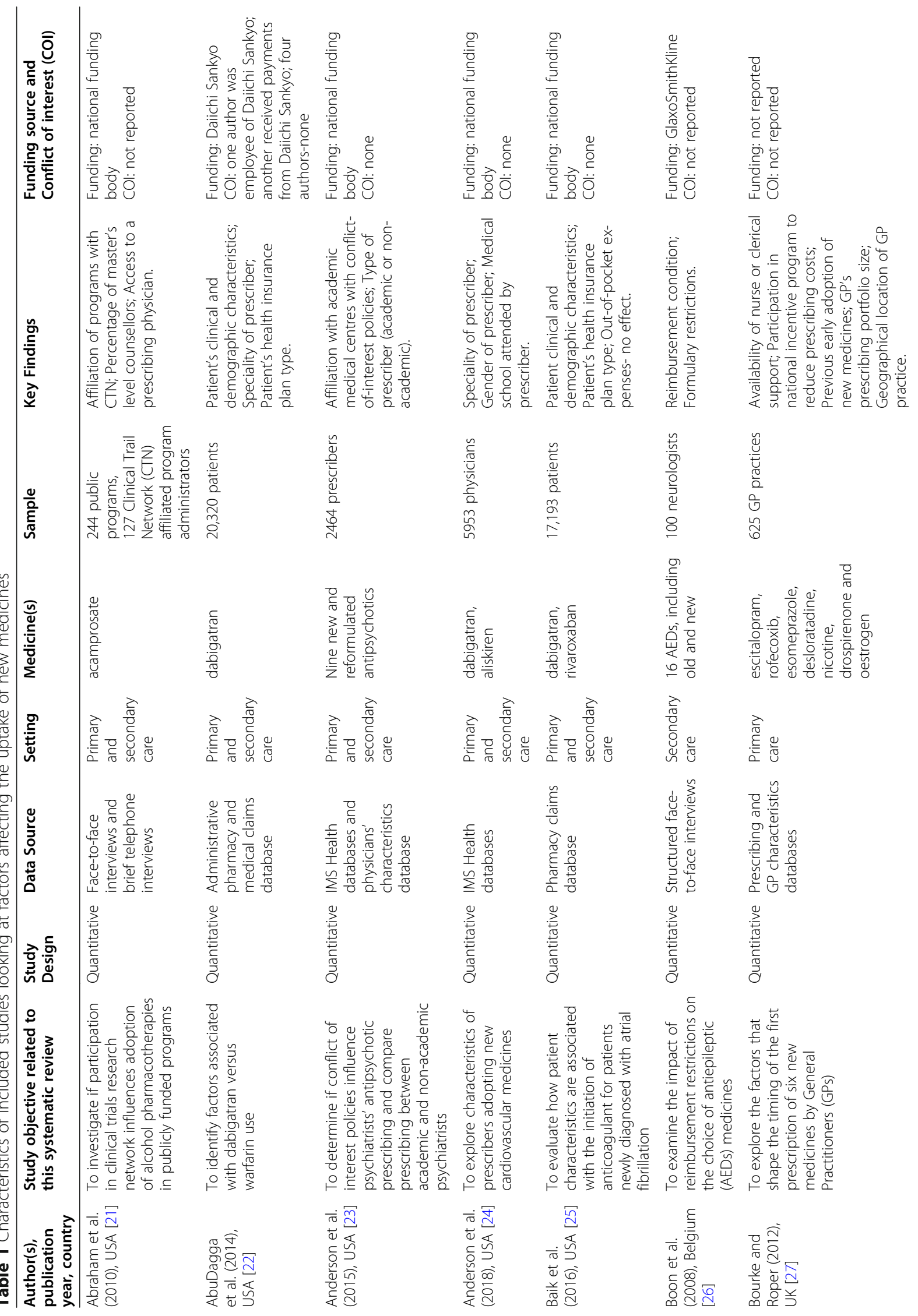




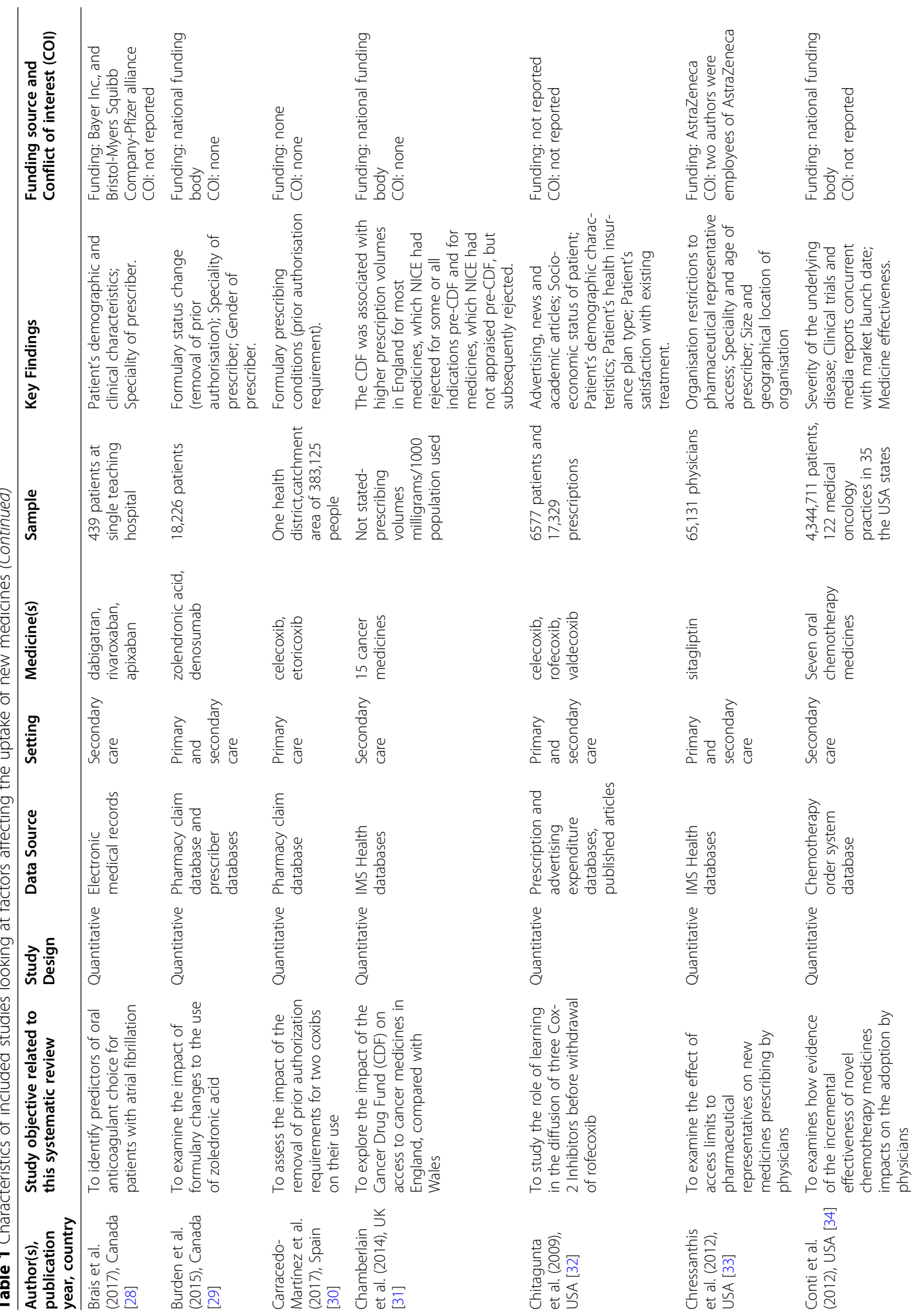




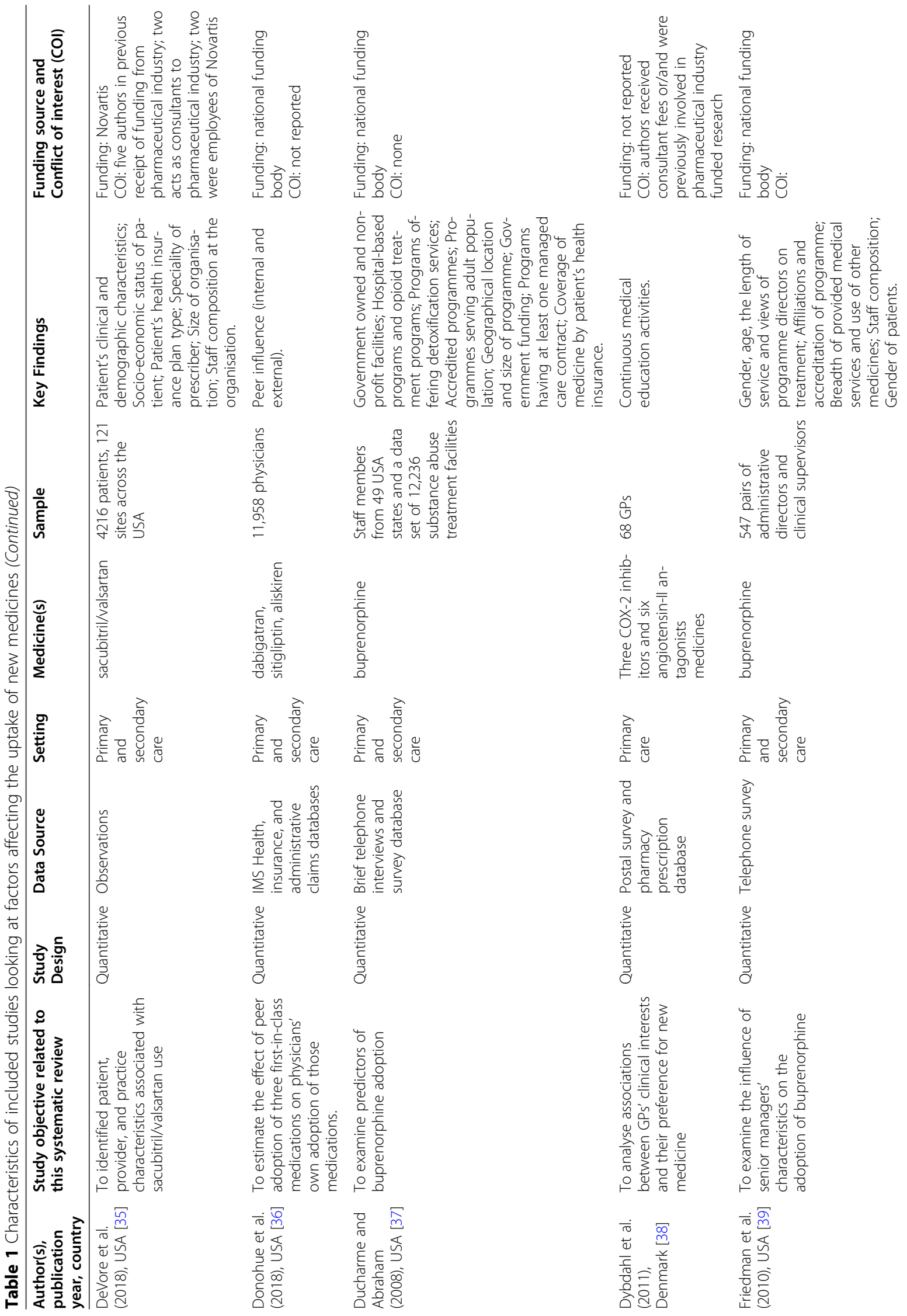




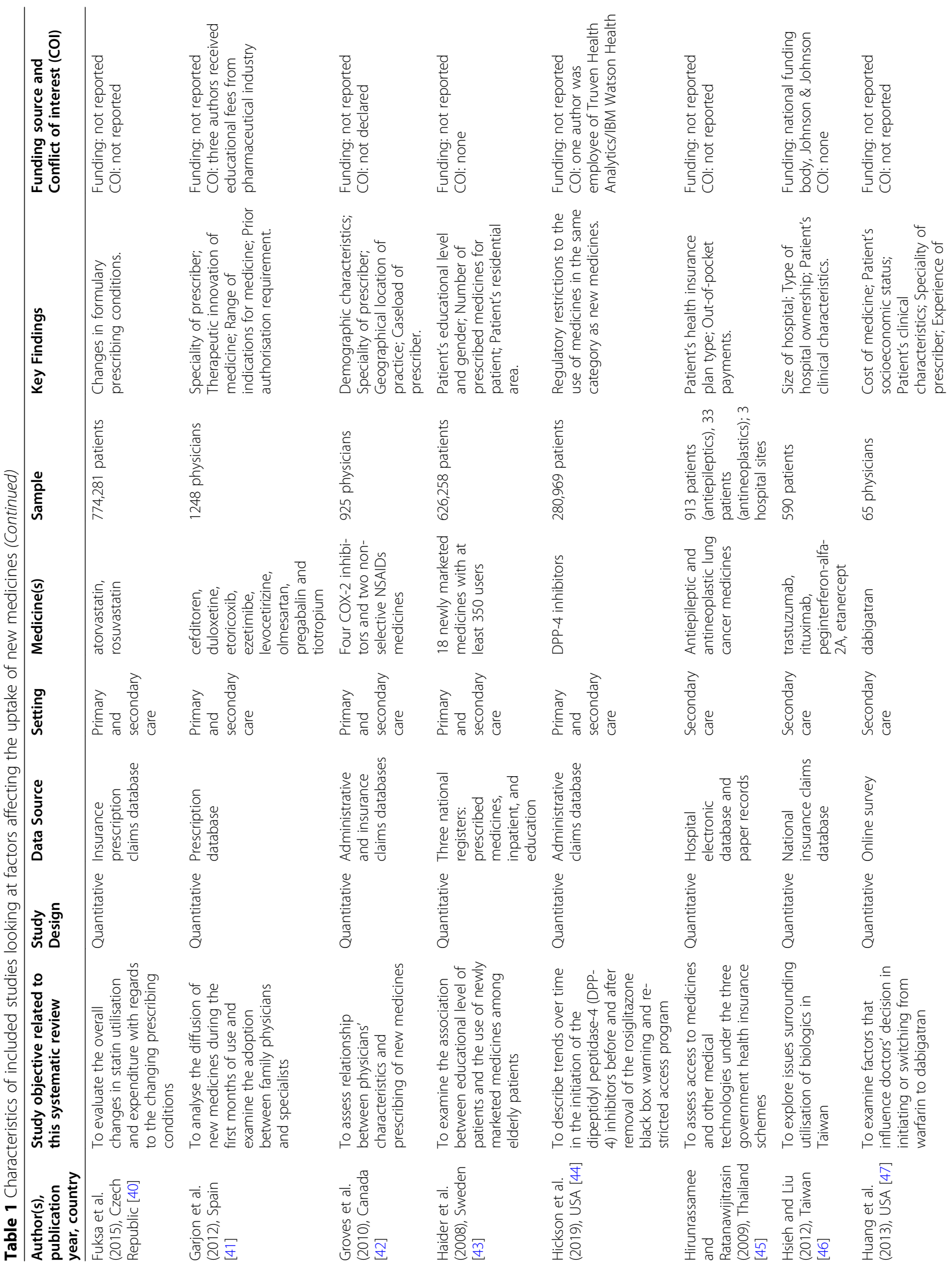




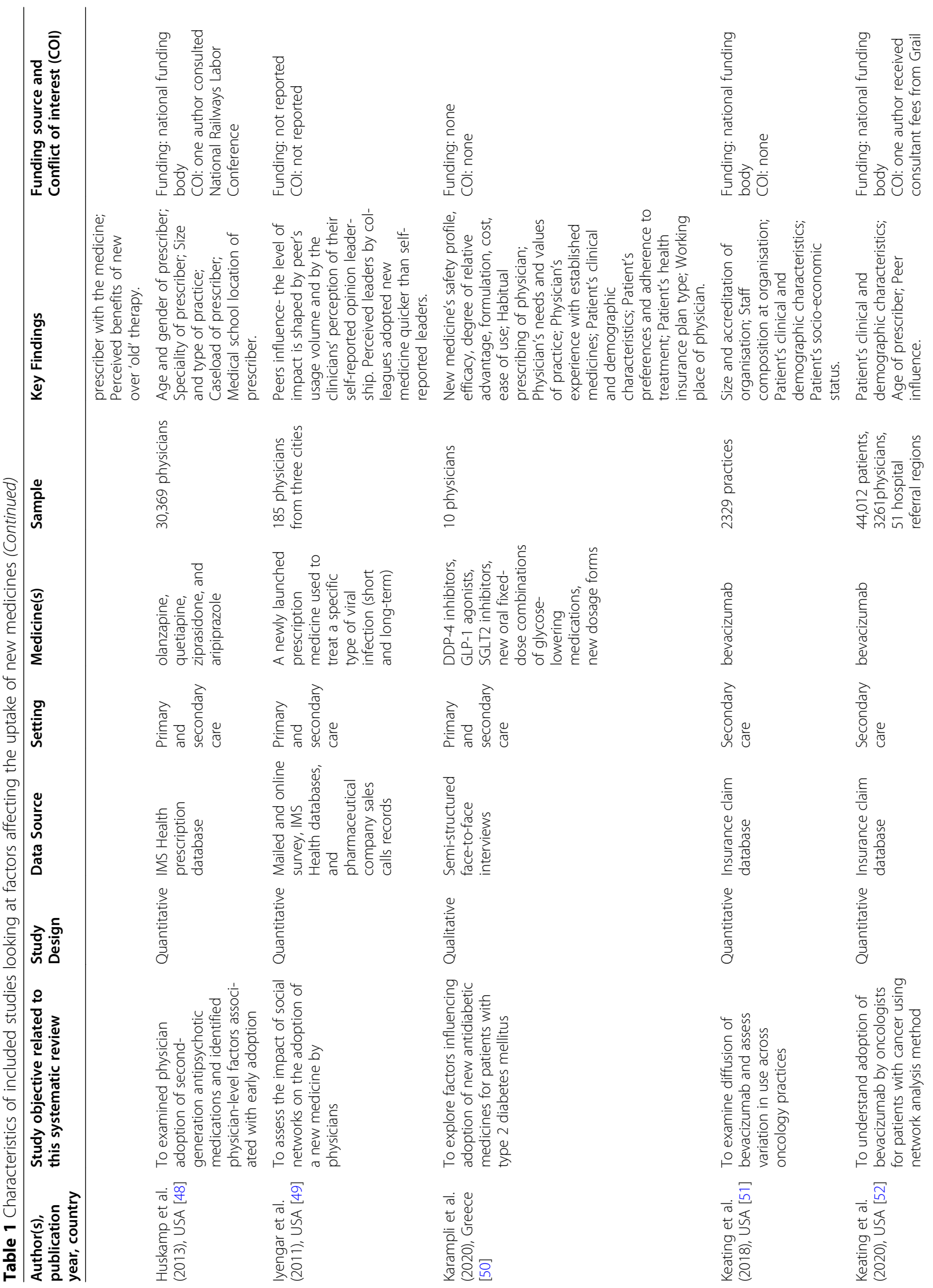




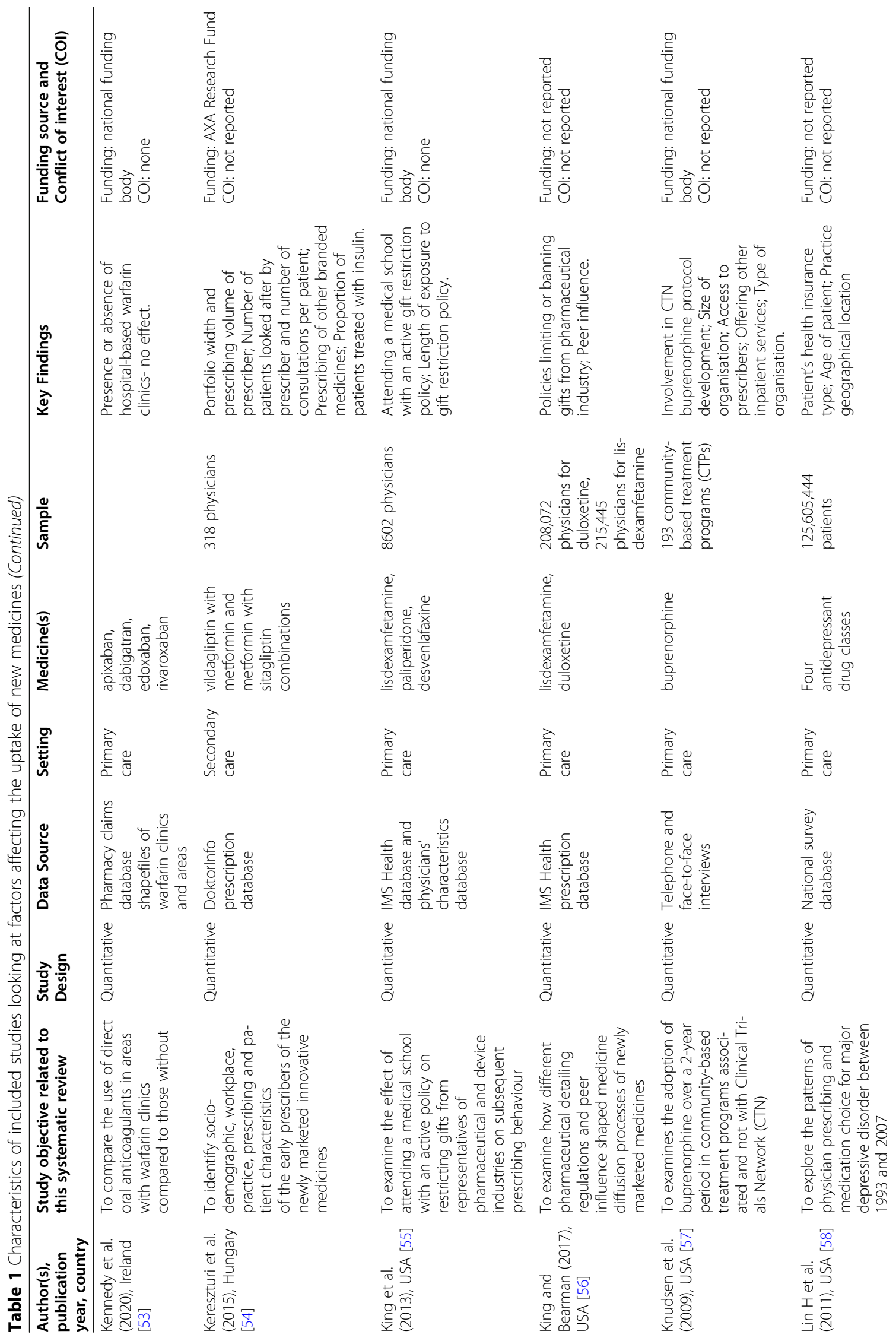




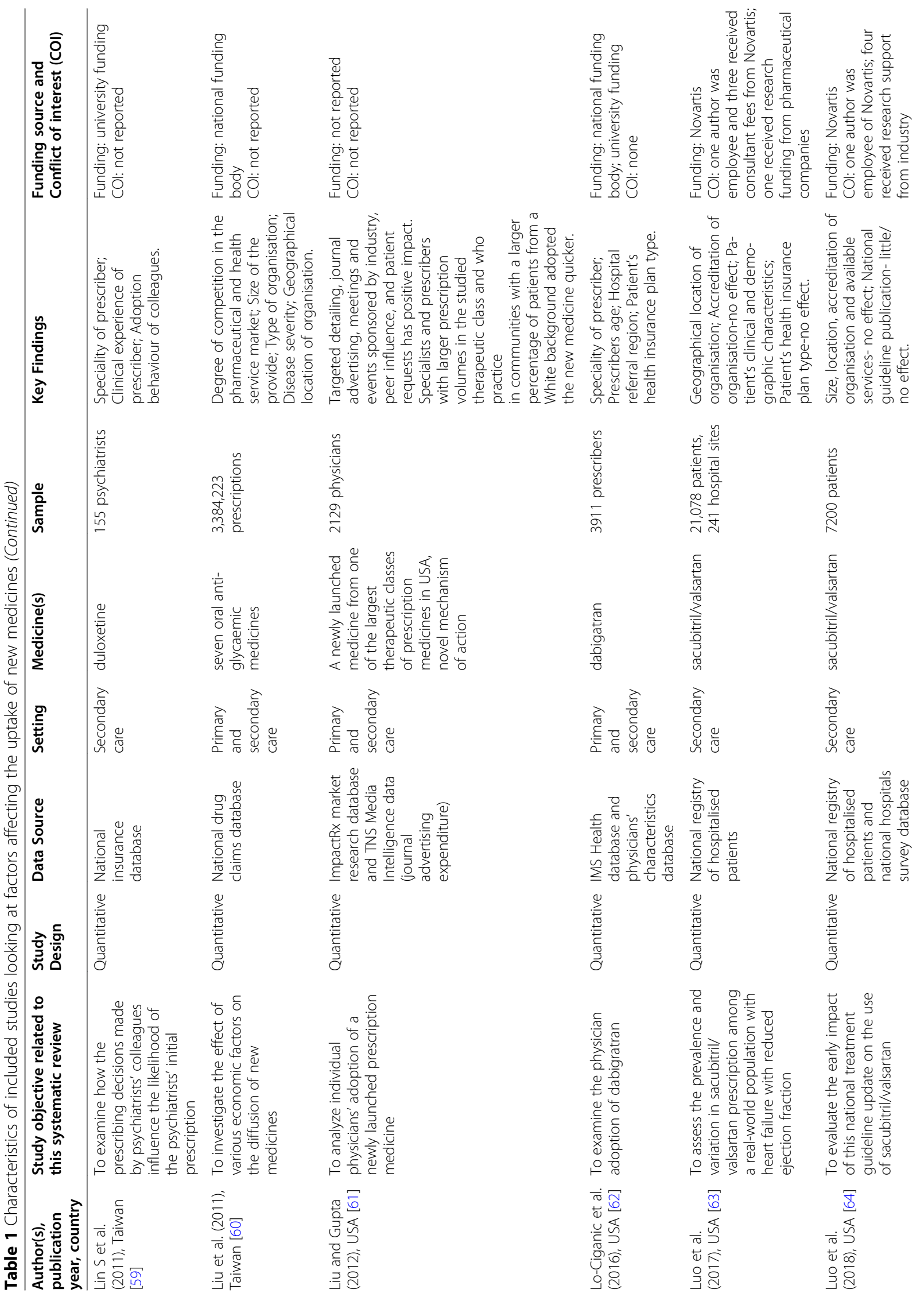




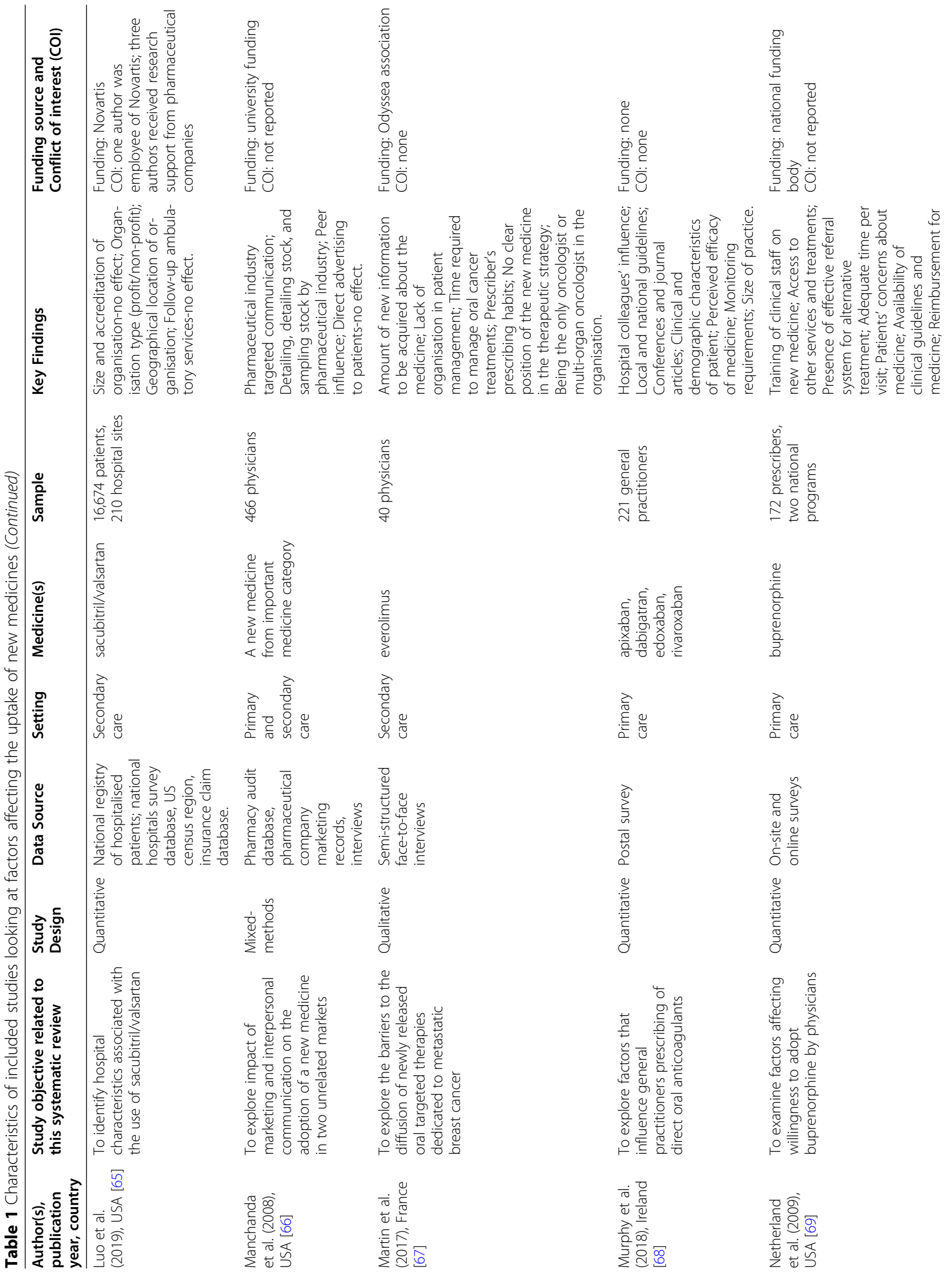




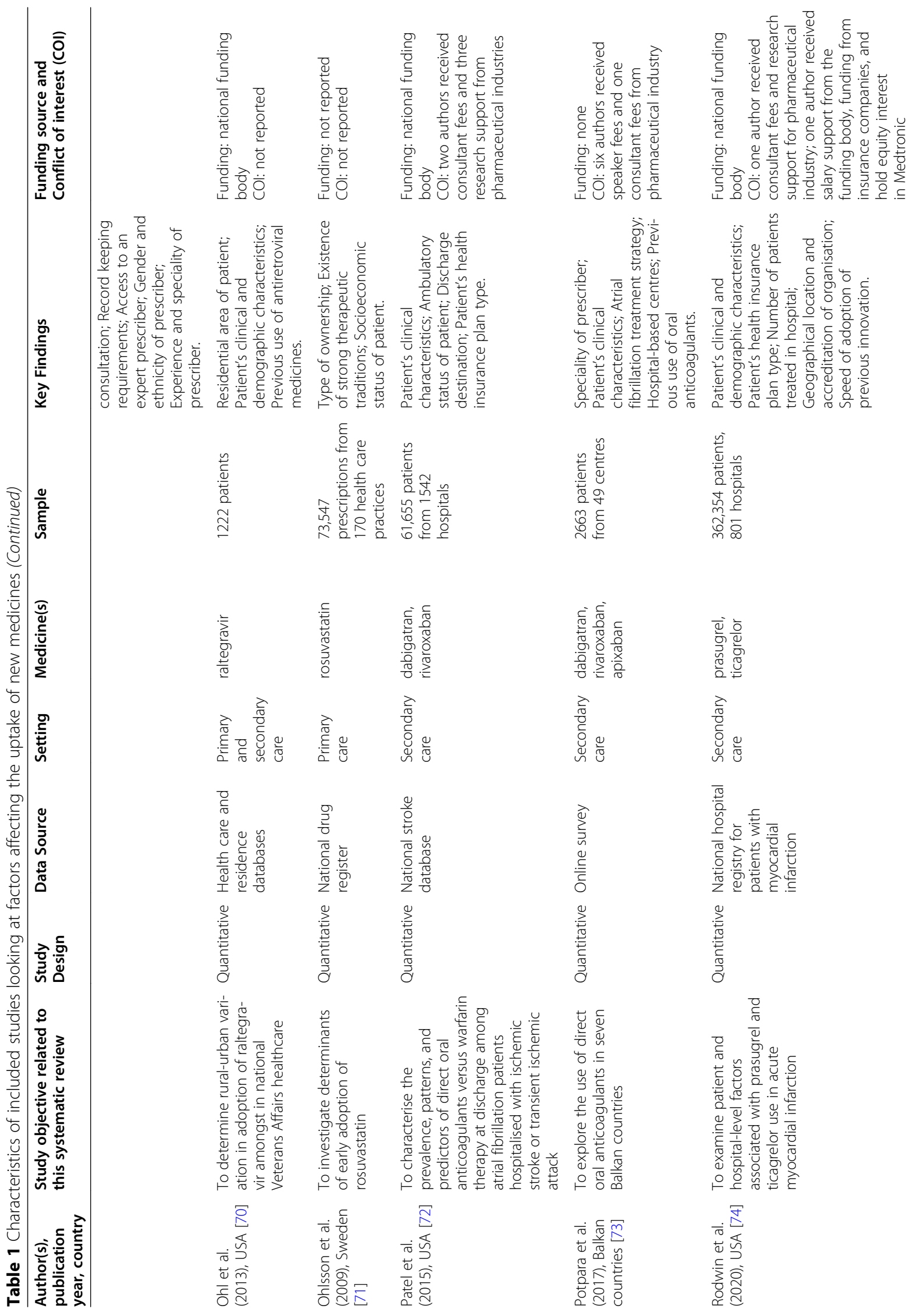




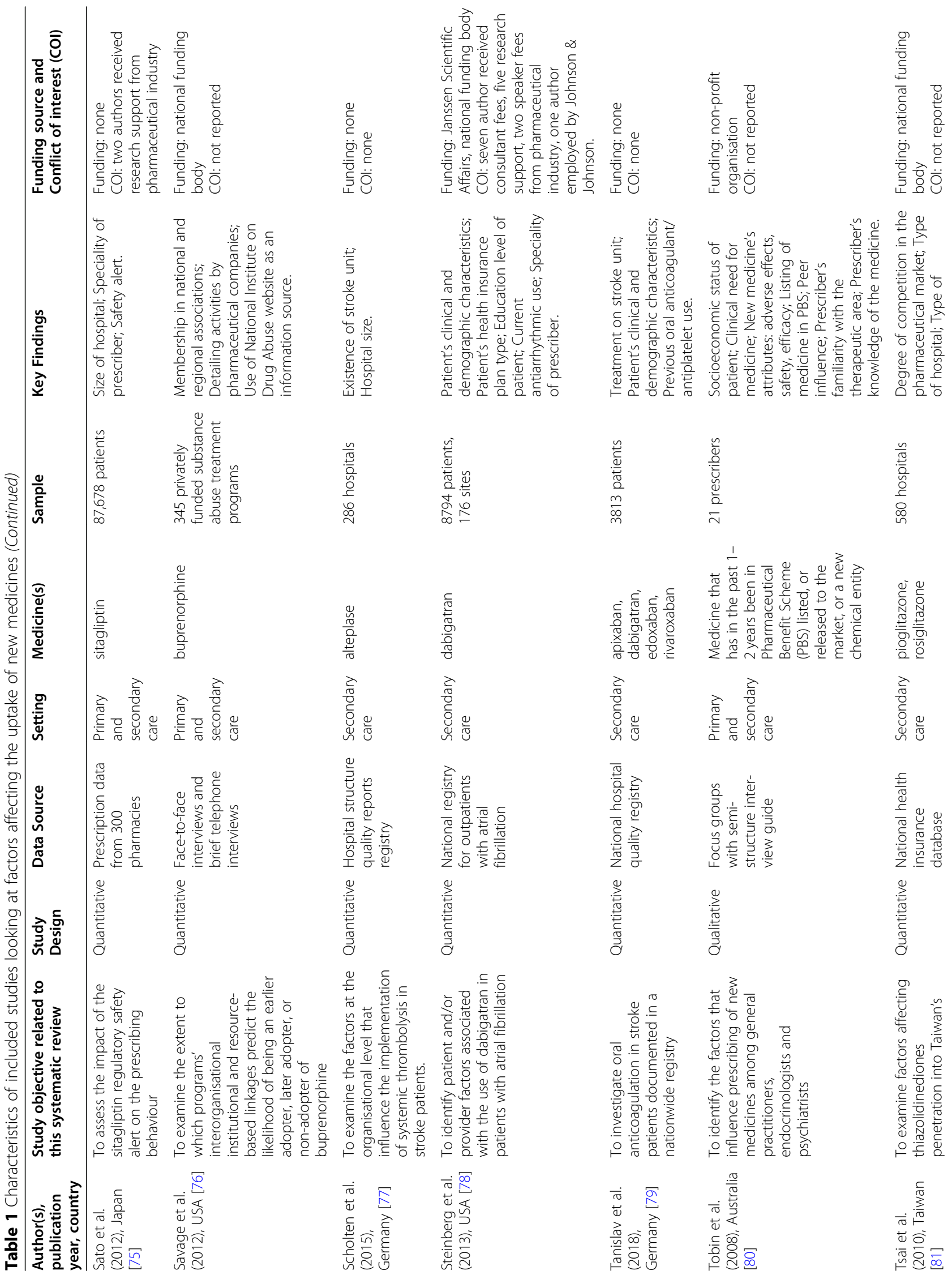




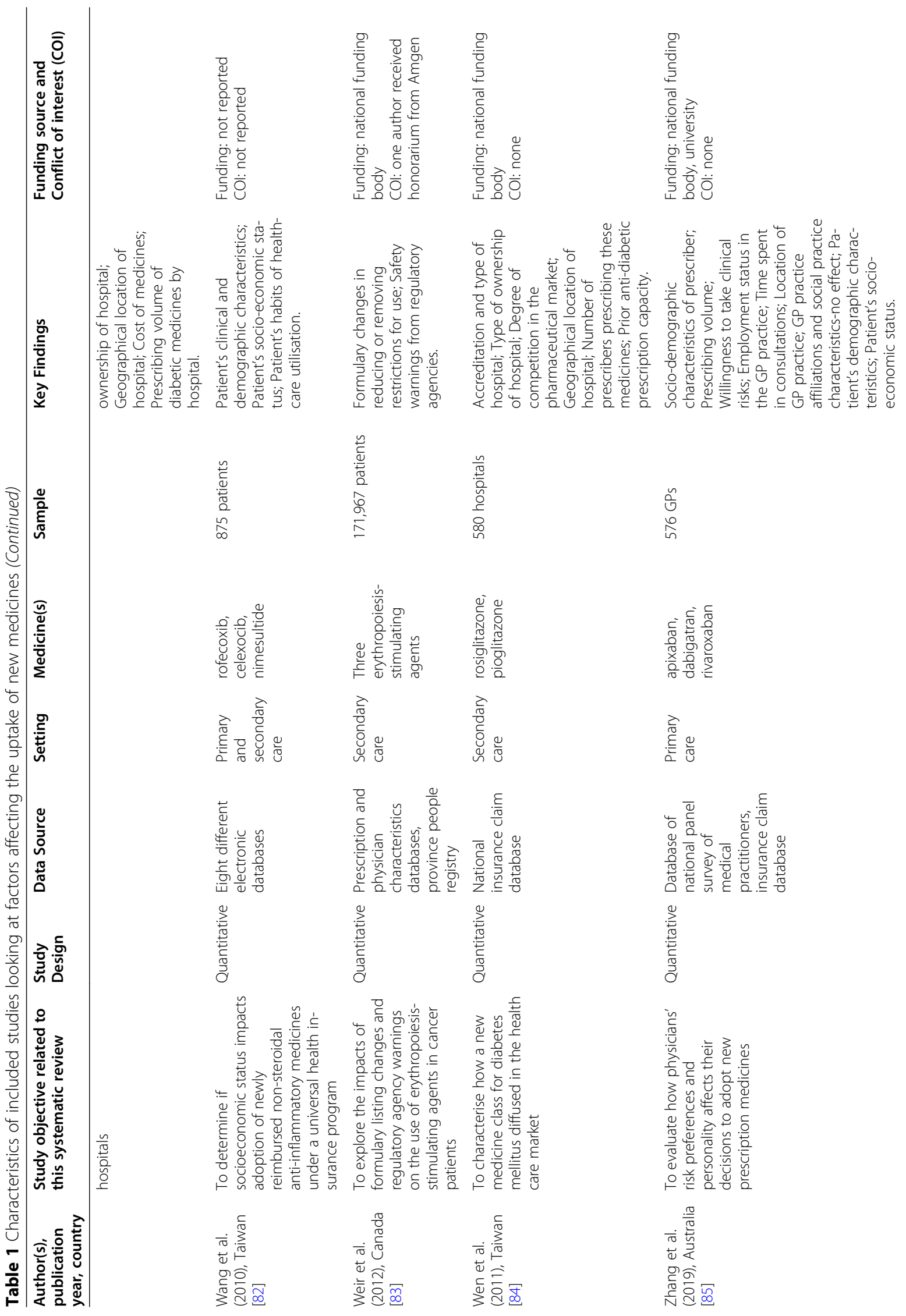




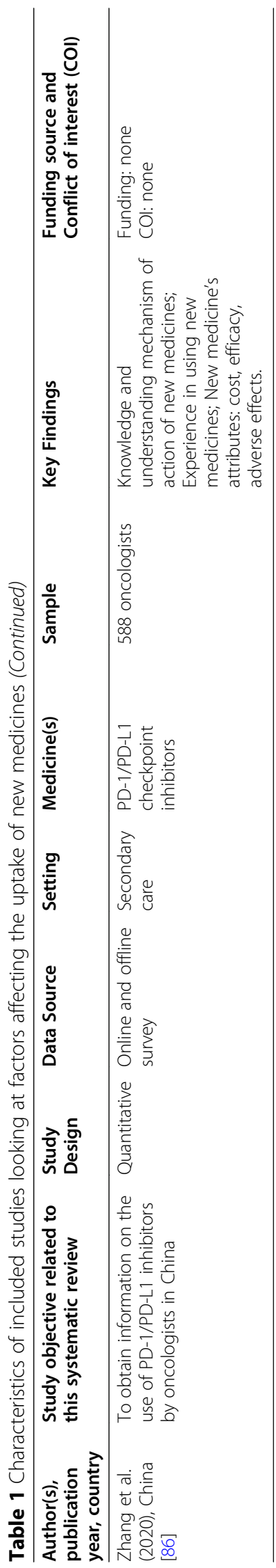


Table 2 Summary of the scores for the 16 criteria used to assess the methodological quality shown for all studies, quantitative and qualitative studies*

\begin{tabular}{|c|c|c|c|c|}
\hline QASTDD tool criteria and study design & Range & Mean & $\begin{array}{l}\text { Standard } \\
\text { deviation }\end{array}$ & $\begin{array}{l}\% \text { maximum of } \\
\text { possible score } \\
\text { achieved }\end{array}$ \\
\hline 1. Explicit theoretical framework (all studies) & $0-3$ & 1.9 & 0.9 & $62 \%$ \\
\hline Quantitative studies & $0-3$ & 1.9 & 0.9 & $62 \%$ \\
\hline Qualitative studies & $1-3$ & 1.7 & 1.2 & $56 \%$ \\
\hline 2. Statement of aims/objectives in main body of report (all studies) & $2-3$ & 2.8 & 0.4 & $93 \%$ \\
\hline Quantitative studies & $2-3$ & 2.8 & 0.4 & $92 \%$ \\
\hline Qualitative studies & 3 & 3 & 0 & $100 \%$ \\
\hline 3. Clear description of research setting (all studies) & $2-3$ & 2.7 & 0.5 & $89 \%$ \\
\hline Quantitative studies & $2-3$ & 2.7 & 0.5 & $89 \%$ \\
\hline Qualitative studies & $2-3$ & 2.7 & 0.5 & $89 \%$ \\
\hline 4. Evidence of sample size considered in terms of analysis (all studies) & $0-3$ & 1.5 & 0.7 & $50 \%$ \\
\hline Quantitative studies & $1-3$ & 1.5 & 0.6 & $49 \%$ \\
\hline Qualitative studies & $0-3$ & 2 & 1.7 & $67 \%$ \\
\hline 5. Representative sample of target group of a reasonable size (all studies) & $1-3$ & 2.2 & 0.5 & $74 \%$ \\
\hline Quantitative studies & $1-3$ & 2.2 & 0.5 & $75 \%$ \\
\hline Qualitative studies & 2 & 2 & 0 & $67 \%$ \\
\hline 6. Description of procedure for data collection (all studies) & $1-3$ & 2.4 & 0.7 & $81 \%$ \\
\hline Quantitative studies & $1-3$ & 2.4 & 0.7 & $81 \%$ \\
\hline Qualitative studies & $2-3$ & 2.7 & 0.6 & $89 \%$ \\
\hline 7. Rationale for choice of data collection tool(s) (all studies) & $0-3$ & 1.7 & 0.9 & $58 \%$ \\
\hline Quantitative studies & $0-3$ & 1.8 & 0.8 & $59 \%$ \\
\hline Qualitative studies & $0-2$ & 0.7 & 1.2 & $22 \%$ \\
\hline 8. Detailed recruitment data (all studies) & $0-3$ & 2.0 & 1.0 & $67 \%$ \\
\hline Quantitative studies & $0-3$ & 2.0 & 1.0 & $68 \%$ \\
\hline Qualitative studies & $0-3$ & 1.7 & 1.5 & $56 \%$ \\
\hline $\begin{array}{l}\text { 9. Statistical Assessment of reliability and validity of measurement tool(s) } \\
\text { (Quantitative studies only) }\end{array}$ & $0-3$ & 0.9 & 1.1 & $29 \%$ \\
\hline $\begin{array}{l}\text { 10. Fit between stated research question and method of data collection } \\
\text { (Quantitative studies only) }\end{array}$ & $2-3$ & 2.8 & 0.4 & $93 \%$ \\
\hline $\begin{array}{l}\text { 11. Fit between stated research question and format and content of data } \\
\text { collection tool e.g. interview schedule (Qualitative studies only) }\end{array}$ & $2-3$ & 2.3 & 0.5 & $75 \%$ \\
\hline 12. Fit between research question and method analysis (all studies) & $2-3$ & 2.9 & 0.3 & $97 \%$ \\
\hline Quantitative studies & $2-3$ & 2.9 & 0.2 & $98 \%$ \\
\hline Qualitative studies & $2-3$ & 2.3 & 0.6 & $78 \%$ \\
\hline 13. Good justification for analytical method selected (all studies) & $0-3$ & 2.4 & 0.8 & $81 \%$ \\
\hline Quantitative studies & $0-3$ & 2.5 & 0.8 & $83 \%$ \\
\hline Qualitative studies & $2-3$ & 2.7 & 0.6 & $89 \%$ \\
\hline 14. Assessment of reliability of analytical process (Qualitative studies only) & $0-2$ & 1.0 & 0.8 & $33 \%$ \\
\hline 15. Evidence of user involvement in design (all studies) & 0 & 0 & 0 & $0 \%$ \\
\hline 16. Strengths and limitations critically discussed (all studies) & $0-3$ & 1.9 & 0.7 & $63 \%$ \\
\hline Quantitative studies & $0-3$ & 1.9 & 0.7 & $63 \%$ \\
\hline Qualitative studies & $1-2$ & 1.3 & 0.6 & $44 \%$ \\
\hline
\end{tabular}

${ }^{*}$ Quantitative studies $n=62$, qualitative studies $n=3$, mixed-methods study $n=1$. As there was only one mixed-methods study, its scores were included in reporting score for all studies and individual scores can be found in Additional file 3, Table S1 
Table 3 Summary of main thematic areas and developed subthemes of factors affecting the uptake of new medicines

\begin{tabular}{|c|c|}
\hline Thematic area & Subthemes \\
\hline \multirow[t]{4}{*}{ Patient-level factors } & Demographic characteristics \\
\hline & Socioeconomic status \\
\hline & Health status \\
\hline & Patient engagement with treatment \\
\hline \multirow[t]{3}{*}{ Prescriber factors } & Socio-demographic characteristics \\
\hline & Scope of expertise \\
\hline & Knowledge and prescribing habits \\
\hline \multirow[t]{5}{*}{ Medicine-level factors } & Efficacy \\
\hline & Safety profile \\
\hline & Cost \\
\hline & Therapeutic innovation \\
\hline & Medicine administrative burden \\
\hline \multirow[t]{7}{*}{ Organizational-level factors } & Ownership status \\
\hline & Teaching status \\
\hline & Size \\
\hline & Location \\
\hline & Available services and resources \\
\hline & Staff composition \\
\hline & Care co-ordination and quality \\
\hline \multirow[t]{6}{*}{ External environment-level factors } & Pharmaceutical detailing \\
\hline & Reimbursement conditions and formulary status \\
\hline & Peer influence (internal and external) \\
\hline & Guidelines \\
\hline & Other information sources \\
\hline & Organization affiliations \\
\hline
\end{tabular}

were a principal or partner in a practice were more likely to use new medicines than employee GPs [85].

\section{Scope of expertise $(n=23)$}

Thirteen studies indicated that specialist prescribers adopted new medicines quicker than their other or primary care colleagues $[22,24,28,33,41,47,48,59,61,62$, $73,78]$ but only three were high-quality $[24,61,78]$. Other medium to high quality studies reported the opposite [29, $42,48,75]$ or no impact [35]. A high-quality study observed the clinical interest of primary care prescribers did not influence new medicine prescribing from the same clinical area [38]. Increasing total prescribing volume [48, $54,85]$ or greater prescribing portfolio breadth $[27,54]$ in medium-quality studies, prescribing multiple medicines for the same condition [84] or larger prescription volume in the same therapeutic class [61] in high-quality studies were suggested to increase adoption of new medicines. Also, a high-quality study observed non-academic prescribers were more likely to use new and reformulated antipsychotics [23].

\section{Knowledge and prescribing habits $(n=10)$}

Medium-quality studies suggested prescribers' previous experience and knowledge of using new medicines increased their use $[47,67-69,80,86]$, whereas a lack of knowledge and confidence delayed or prevented use [50, 67]. Some prescribers commented that an overwhelming amount of new information for new medicines prescribing discouraged their use [67]. A high-quality study observed that continuing medical education activities supported prescribing of new medicine in one of two studied therapeutic areas [38]. In medium-quality studies, prescribers classed as early adopters in the past [27], or more likely to take clinical risks [85], or spend less time in patient consultations [85] tended to use new medicines quicker.

\section{Medicine-level factors \\ Efficacy $(n=6)$}

Some prescribers stated relative effectiveness of a new medicine influenced their prescribing decisions in medium-quality studies $[50,68,69,80,86]$. A highquality study, focusing on novel chemotherapies, 
Table 4 Summary of factors affecting the uptake of new medicines referred to in the reviewed studies

\begin{tabular}{|c|c|c|c|c|c|}
\hline Identified factor & $\begin{array}{l}\text { Number of studies } \\
\text { referred to the factor }\end{array}$ & $\begin{array}{l}\text { As } \\
\text { facilitator }\end{array}$ & $\begin{array}{l}\text { As } \\
\text { barrier }\end{array}$ & $\begin{array}{l}\text { No } \\
\text { impact }\end{array}$ & Citations \\
\hline \multicolumn{6}{|l|}{ Patient factors } \\
\hline Age (younger) & 18 & 11 & 4 & 3 & $\begin{array}{l}{[22,25,28,32,35,51,52,58,60,63,} \\
70-74,78,79,82,85]\end{array}$ \\
\hline Gender (male) & 12 & 4 & 1 & 7 & {$[22,28,29,58,60,71-74,78,79,82]$} \\
\hline Ethnicity (White) & 10 & 6 & 1 & 3 & {$[25,35,51,58,61,70,72,74,78,82]$} \\
\hline Education level (higher) & 5 & 4 & & 1 & {$[32,35,43,78,82]$} \\
\hline Income (higher) & 11 & 11 & & 1 & {$[22,25,32,45,47,50,51,71,80,82,85]$} \\
\hline Insurance (private or more comprehensive) & 9 & 9 & & & {$[22,25,35,45,58,62,72,74,78]$} \\
\hline Residential area (urban or more affluent) & 3 & 3 & & & {$[43,70,85]$} \\
\hline $\begin{array}{l}\text { Health condition (more severe \& } \\
\text { comorbidities) }\end{array}$ & 13 & 5 & 8 & & $\begin{array}{l}{[22,28,34,35,46,51,63,72-74,78,79,} \\
82]\end{array}$ \\
\hline Polypharmacy & 9 & 3 & 4 & & {$[22,25,28,43,72,73,79]$} \\
\hline $\begin{array}{l}\text { Patient satisfaction, adherence to current } \\
\text { therapy \& monitoring }\end{array}$ & 4 & 4 & & & {$[32,47,50,80]$} \\
\hline Response to current therapy (poor) & 3 & 3 & & & {$[47,70,80]$} \\
\hline Patients request \& therapy preferences & 5 & 5 & & & {$[47,50,61,69,80]$} \\
\hline \multicolumn{6}{|l|}{ Prescriber factors } \\
\hline Age (younger) & 7 & 4 & 2 & 3 & {$[27,29,33,42,48,53,62]$} \\
\hline Gender (male) & 6 & 4 & & 2 & {$[23,29,42,48,62,85]$} \\
\hline $\begin{array}{l}\text { Graduating from a top-20 medical or foreign } \\
\text { school }\end{array}$ & 3 & 3 & & 2 & {$[23,48,62]$} \\
\hline Principal or partner GP & 1 & 1 & & & [85] \\
\hline Specialist or secondary care prescriber & 16 & 13 & 4 & 1 & $\begin{array}{l}{[22,24,28,29,33,35,41,42,47,48,59,} \\
61,62,73,75,78]\end{array}$ \\
\hline Non-academic prescriber & 1 & 1 & & & [23] \\
\hline $\begin{array}{l}\text { Greater prescribing volume or portfolio } \\
\text { breadth }\end{array}$ & 5 & 5 & & & {$[27,48,54,61,85]$} \\
\hline Knowledge of new medicine & 7 & 7 & & & {$[47,50,67-69,80,86]$} \\
\hline Continuing medical education activities & 1 & 1 & & & {$[38]$} \\
\hline Early adopter in the past & 1 & 1 & & & [27] \\
\hline $\begin{array}{l}\text { Taking clinical risks \& spending less time in } \\
\text { consultations }\end{array}$ & 1 & 1 & & & [85] \\
\hline \multicolumn{6}{|l|}{ Medicine factors } \\
\hline Efficacy & 6 & 6 & & & {$[34,50,68,69,80,86]$} \\
\hline Safety concerns (adverse \& long-term effects) & 6 & & 6 & & {$[44,47,50,68,80,86]$} \\
\hline Interactions with food/medicines (less) & 3 & 3 & & & {$[47,68,80]$} \\
\hline High unit cost & 5 & 5 & & 3 & {$[47,50,68,80,86]$} \\
\hline Therapeutic innovation & 5 & 5 & & & {$[41,48,50,60,80]$} \\
\hline Ease of use \& administration & 4 & 3 & 1 & 1 & {$[47,50,69,80]$} \\
\hline Reduced monitoring \& clinic visits & 2 & 2 & & & {$[47,68]$} \\
\hline \multicolumn{6}{|l|}{ Organizational factors } \\
\hline Ownership status (private) & 10 & 7 & 2 & 1 & {$[21,37,46,57,60,64,65,71,81,84]$} \\
\hline Teaching status & 8 & & 1 & 7 & {$[54,63-65,72-74,77]$} \\
\hline Size (larger) & 17 & 11 & 3 & 3 & $\begin{array}{l}{[33,35,37,46,48,51,57,60,65,68,72,} \\
75-77,81,82,84]\end{array}$ \\
\hline Location (more populated) & 10 & 3 & 3 & 5 & {$[27,42,57,63,71-73,77,84,85]$} \\
\hline
\end{tabular}


Table 4 Summary of factors affecting the uptake of new medicines referred to in the reviewed studies (Continued)

\begin{tabular}{|c|c|c|c|c|c|}
\hline Identified factor & $\begin{array}{l}\text { Number of studies } \\
\text { referred to the factor }\end{array}$ & $\begin{array}{l}\text { As } \\
\text { facilitator }\end{array}$ & $\begin{array}{l}\text { As } \\
\text { barrier }\end{array}$ & $\begin{array}{l}\text { No } \\
\text { impact }\end{array}$ & Citations \\
\hline Availability of supportive services & 11 & 7 & & 4 & {$[27,35,37,39,53,57,65,69,76,77,79]$} \\
\hline Limited consultation time & 2 & & 2 & & {$[67,69]$} \\
\hline $\begin{array}{l}\text { Number of specialists, nurses, or healthcare } \\
\text { professionals (higher) }\end{array}$ & 8 & 8 & & & {$[21,35,39,48,51,57,59,67]$} \\
\hline Care co-ordination (fragmented) & 2 & & 2 & & {$[67,69]$} \\
\hline \multicolumn{6}{|l|}{ External environment factors } \\
\hline Pharmaceutical detailing & 11 & 11 & & 1 & {$[23,33,50,55,61,66,68,76,80]$} \\
\hline Formulary or reimbursement restrictions & 10 & & 10 & & {$[23,26,29-31,37,40,50,80,83]$} \\
\hline Peer influence (internal \& external) & 14 & 14 & & & $\begin{array}{l}{[36,49,50,52,56,59,61,64,66,68,71,} \\
80,81,84]\end{array}$ \\
\hline $\begin{array}{l}\text { Recommended by guideline (international, } \\
\text { national, or local) }\end{array}$ & 6 & 5 & & 1 & {$[50,64,67-69,86]$} \\
\hline Scientific literature, websites, \& conferences & 6 & 6 & 1 & & {$[32,34,50,68,76,80]$} \\
\hline Organizational affiliations & 6 & 4 & 2 & & {$[21,39,50,51,57,76]$} \\
\hline
\end{tabular}

suggested that perceived better quality rather than incremental effectiveness influenced new medicine use [34].

\section{Safety profile $(n=9)$}

Some prescribers reported that concerns over adverse effects $[47,50,68,80,86]$ and unknown long-term risks [50] discouraged prescribing new medicines. Less interactions with other medicines or food [47, 68, 80], and less reported adverse effects [47] compared to existing treatments, encouraged uptake. All were medium-quality studies. Medium to high quality studies observed that national safety reports, e.g., Food and Drug Administration, highlighting safety concerns contributed to hesitancy of some prescribers to use new medicines [44, 83]. Also, a high-quality study suggested that scientific articles [32] rather than safety alerts influenced prescribing behaviours as safety concerns would be first reported in the scientific literature. Another medium-quality study suggested safety concerns with an existing class of medicines encouraged prescribers to use new medicines from a therapeutically different class [44].

\section{Cost $(n=9)$}

Some prescribers reported a higher unit cost of a new medicine over existing therapy was a barrier for its use $[47,50,68,80,86]$. However, a proportion of prescribers did not consider a medicine's cost in their prescribing decisions $[47,68,86]$. The unit cost of the new medicine was perceived differently by prescribers and patients. Patients appeared willing to pay more if the new medicine was in their best interest [80]. In contrast, prescribers considered the patient's ability to pay out of pocket costs $[22,45,47$, $50,71,82]$, which could affect patients' adherence to therapy and affordability of future prescriber's visits [50]. Some prescribers also discussed their role in containing spending of social insurance, although others thought cost-savings to public spending was not a prescriber's job [50]. Only two studies were high-quality [71, 82].

\section{Therapeutic innovation $(n=5)$}

Two studies suggested new medicines [41] or reformulations [48], perceived as having therapeutic innovation, were adopted quicker than medicines without. Another study indicated the availability of more medicines within the same therapeutic category (i.e., higher competition) had a negative impact on new medicines entering the same category use [60]. Some prescribers reported considering a new medicine's relative clinical benefits other than safety, efficacy, or cost over existing treatment [50, 80]. For instance, a positive effect on patient's weight, comorbidities, and cardiovascular protection by new antidiabetic medicines [50]. All studies were medium quality.

\section{Medicine administrative burden $(n=5)$}

Some prescribers stated the ease of administration [80] or use [50] of the medicine facilitated its uptake. Another study observed that increased complexity of taking a new medicine, e.g., twice a day, was a barrier to a minority of prescribers [47]. The majority of prescribers in the case of oral anticoagulants reported reduced monitoring or clinic visits encouraged their use [47, 68]. Also, concerns about difficulty to initiate new medicines negatively affected the willingness of some prescribers to use them, especially if they were less experienced [69]. All studies were medium quality.

\section{Organizational-level factors \\ Ownership status $(n=10)$}

Four high [37, 71, 81, 84] and three medium [57, 60, 65] quality studies suggested private, rather than public 
organizations, were more likely to use new medicines. Amongst private organizations, for-profit treatment programs were more likely to offer new medicines [37, 57]. In contrast, medium to high quality studies observed public organizations having greater use of new medicines $[21,46]$ or the ownership status did not influence the uptake [64].

\section{Teaching status $(n=8)$}

Six medium studies [24, 63-65, 72, 73] and one high [77] quality study observed no difference in the uptake of new medicines between teaching and non-teaching hospitals. One medium-quality study, however, suggested a lower likelihood of new medicine use at a teaching hospital [74].

Size $(n=17)$

Six high $[35,37,46,81,82,84]$ and five medium $[48,51$, $57,60,68]$ quality studies indicated larger hospitals or practices were more likely to use new medicines. Other medium to high quality studies observed it for smaller $[33,75]$ or medium size [77] organizations. Also, three medium-quality studies suggested organization size did not influence the uptake $[65,72,76]$.

\section{Location $(n=16)$}

In some medium to high quality studies, organizations in urban areas $[27,57,73]$, rural locations $[42,84]$, or in areas with fewer GPs [85] were observed to have a higher use of new medicines. Five medium to high quality studies reported geographical location having no impact on the uptake $[27,63,71,72,77]$. Also, nine studies reported regional variation in prescribing of new medicines $[46,60,63,65,72,74,81,85]$.

\section{Available services and resources $(n=13)$}

In some cases, organizations providing, or having access to, related supportive services were more likely to adopt new medicines [37, 39, 57, 69, 76, 77, 79]; two were of high [69, 77] and one low [79] quality. For instance, detoxification, mental health services, or substance abuse counselling services for buprenorphine [37, 57, 69, 76] or stroke units for alteplase and direct oral anticoagulants $[77,79]$ were reported to facilitate the uptake. In other cases, supporting services such as the availability of heart failure clinics [35] or follow-up after hospitalisation [65] for sacubitril/valsartan, availability of hospitalbased anticoagulant monitoring clinics for direct oral anticoagulants [53], or presence of dispensing services within general practices [27] had no impact. Also, prescribers reported lack of adequate time per patient visit acted as a barrier $[67,69]$, especially for less experienced prescribers [69]. Furthermore, some primary care clinicians suggested secondary care colleagues had more learning opportunities available (e.g., participation in clinical trials, education, and learning, access to more patients) supporting new medicine use [50].

\section{Staff composition ( $n=9$ )}

Medium-quality studies indicated that lack of specialist prescribers was a barrier to new medicine use [21, 39, $48,51,57,67]$. For instance, organizations with more qualified staff [21] and GPs with hospital experience [59] were reported to adopt some of the studied medicines quicker. A high-quality study reported that organizations with higher numbers of nurses, and healthcare professionals with a generalist medical education, were more likely to use new medicines and the number of specialist prescribers had no influence [35]. Another mediumquality study reported the presence of clerical and nursing staff to have limited to no impact on the uptake [27].

\section{Care co-ordination and quality $(n=3)$}

Some prescribers suggested that lack of organization and fragmentation in the provision of patient care [67], and non-clinical activities of care co-ordination, such as additional record-keeping requirements [69] were barriers to new medicine use. A study looking at heart failure treatment observed a lower uptake of a new medicine within hospitals scoring higher on non-heart failure service quality measures [65]. All studies were medium quality.

\section{External environment-level factors \\ Pharmaceutical detailing $(n=11)$}

The pharmaceutical industry was seen to promote awareness of new medicines through pharmaceutical detailing (pharmaceutical marketing aimed at prescribers) and indirectly through conferences, educational events, advertisements in academic and professional journals $[50,61,80]$. Prescribers in medium-quality studies had mixed views on its impact on their prescribing decisions $[50,68,80]$ with some reporting pharmaceutical representatives as one of their main information sources about new medicines [50]. Three studies in USA indicated that current and/or past detailing with or without distribution of free samples had a positive impact on new medicine uptake [61, 66, 76]; two studies were high-quality [61, 66]. Also, organizations or areas with restricted access to pharmaceutical detailing or marketing regulation policies in place (e.g., ban of gifts, disclosure policy) had lower and slower uptake of new medicines $[23,33,66]$, especially among primary care prescribers [33]; two studies were high-quality [23, 66]. A high quality study suggested gift restrictions having a greater negative impact than disclosure policies [66]. Another high-quality study indicated that prescribers completing training at medical schools with active policies restricting access to the pharmaceutical industry were 
less likely to use new medicines [55]. A medium-quality study suggested that prescribers with very low access to pharmaceutical detailing were slower in changing their prescribing behaviour when negative information about new medicines was released [33]. Lastly, a high-quality study reported direct-to-consumer advertising aimed at patients had no influence on the uptake [66].

\section{Reimbursement conditions and formulary status $(n=13)$}

Nine studies suggested that reimbursement conditions for a medicine influenced the use of new medicines [23, $26,29-31,40,50,80,83]$; two high $[29,83]$ and one low [30] quality studies. Formulary or reimbursement restrictions $[50,80]$ or cost-control regulatory measures $[23,50]$ were suggested to have negative impact on new medicine use. Removing reimbursement restrictions such as a requirement of prior authorisation [29, 30], specialist use only in secondary care [40], only as second-line therapy $[26,83]$, or providing reimbursement for medicines excluded from a national formulary [31] were suggested to support new medicine use. The inclusion of new medicines in formularies (e.g., public insurance, regional, local, national) was reported to facilitate their use $[37,80]$ with one study being highquality [37]. Also, medium to high quality studies suggested financial incentives to reduce prescribing costs had limited to no impact on the uptake of new medicines already included in formularies [27, 34].

\section{Peer influence (internal and external) $(n=14)$}

Some prescribers indicated that their peers' adoption of new medicines positively influenced their prescribing behaviour of new medicines in eight high $[49,52,56,61$, $66,71,81,84]$ and five medium [36, 50, 59, 68, 80] quality studies. Also, four high-quality studies suggested adoption of new medicines by prescribers after approval was even greater if their peers were early adopters [52, $71,81,84]$. Four medium-quality studies suggested peers from secondary care or specialist areas influenced primary care prescribers $[50,59,68,80]$. Some prescribers stated that other colleagues, opinion leaders, and experts influenced the use of new medicines [50, 59, 64, 80]. One high-quality study indicated peer influence being the greatest from month four of the medicine's launch until month 17 [66]. Another high -quality study observed that peer influence had a greater impact in the states of the USA with policies restricting pharmaceutical marketing [56].

\section{Guidelines $(n=6)$}

Guidelines (local, national, or international) were indicated to influence prescribing decisions of some prescribers [50,68, 69, 86], especially of less experienced $[50,68,69,86]$. Some prescribers reported absence of guidelines prevented [86] or delayed [50] prescribing new medicines till a guideline was released. In one study some prescribers suggested difficulties in determining the position for the new medicine within a clinical pathway was a barrier for the uptake [67]. Contrastingly, one study reported the publication of national guidelines had no impact on the rate of uptake of the studied new medicine [64]. All studies were medium quality.

\section{Other information sources $(n=6)$}

Some prescribers in medium to high quality studies reported conferences, medical or news articles, scientific societies' websites, or clinical trial reports discussing new medicines as having impact on prescribing decisions [32, $34,50,68,76,80]$. A high-quality study, looking at cyclooxygenase- 2 inhibitors, suggested that medical articles discouraged prescribers to use new medicines, but news articles and media reports encouraged it [32]. Another high-quality study, looking at oral chemotherapy agents, observed that clinical trials and media reports published around the Food and Drug Administration (in USA) approval date had positive impact on uptake [34]. Some prescribers reported scientific literature having greater influence in prescribing decisions than information gathered through social professional networks [50] or news media [34]. Also, prescribers using national research websites were suggested to use new medicines earlier [76].

\section{Organization affiliations $(n=6)$}

Three studies indicated organizations' participation in research networks having positive impact on new medicines use [21, 50, 57]. This was attributed to an organization's experience with treatment protocols and exposure to the process of implementing new treatments. Also, organizational links with professional associations were reported to increase the likelihood of being an early adopter in the case of buprenorphine [76]. However, two studies suggested treatment programs affiliated with medical health centres had the same or slower adoption rates than the independent ones [39, 51]. All studies were medium quality.

\section{Discussion}

\section{Summary of evidence}

This systematic review has identified a broad range of factors affecting the uptake of new medicines within healthcare organizations. The identified factors were grouped into patient, prescriber, medicine, organizational, and external environment factors, as per the Chaudoir et al. [7] framework, and overlapped with the Consolidated Framework for Implementation Research (CFIR) [87]. They had a varied impact on the uptake of the different studied new medicines. 
Our review findings, differently from earlier reviews [8$10]$, indicated the presence of patient influence on the uptake of new medicines. Patients were reported to influence prescribing decisions through their interest in or request for new medicines, satisfaction with current treatment, and therapy preferences. However, only a small number of studies reported patient influence and further research is required to establish its relative importance in the uptake of new medicines. Also, reviewed studies did not explore the impact of patient involvement in decision-making, availability of patient choice, and patient-clinician relationship, which are suggested to influence implementation of health innovations [7, 87, 88]. Understanding of these factors could offer explanation for why new medicines are used with some patient types but not others, as established in the reviewed studies.

In our review, high-quality studies indicated that patients with a higher education level were more likely to receive new medicines. This was in contrast to Lubloy's review findings based on one study [10] and not reported in the other two earlier reviews [8,9]. Patient education level has been associated with health education, literacy, and behaviours [89], potentially translating into the level of patient influence on new medicine use. Also, patient education level is linked with patient income [90]. As in Lubloy's review [10], patients with higher income (able to pay out-of-pocket costs) and more comprehensive insurance plans were observed to have greater access to new medicines. This was more predominant in countries without universal health coverage, e.g., USA, but was also present in countries with universal health coverage requiring co-payments from patients, e.g., Taiwan. More studies are needed to explore the impact of patient income on new medicines use in countries with a publicly funded national health service, e.g. UK.

Another important finding was the impact of new medicine cost to healthcare organisations and patients on its uptake. Differently to previous reviews [8-10], our review findings indicated that the high cost of a new medicine was a barrier for the uptake, although to a varied extent. Increasing costs and expenditure on medicines and limited available funding to healthcare services is anticipated to influence uptake of high-cost new medicines [90]. Also, none of the reviewed studies considered the overall costs of new medicines compared to the established therapy (e.g., associated monitoring cost) or health economics (e.g., direct health costs), which could offer explanation to observed geographical variation and restrictions of new medicine use in the reviewed studies.

Our review findings indicated that formulary or reimbursement restrictions influence the uptake of new medicines, which was not reported in earlier reviews [8-10]. The purpose of formulary and reimbursement restrictions is to ensure evidence-based and cost-effective prescribing. These could be used as a cost-control measure for high-cost new medicines, limiting their use. Earlier reviews did not report on impact of guidelines [10] or concluded they had no [8] or varied impact [9]. Our review findings suggest that guidelines have an impact on the uptake. Inclusion of a new medicine in local or national guidelines establishes a new medicine's place in existing clinical pathways and provides assurance to prescribers that they follow the best practice.

Lastly, the review findings reaffirmed that prescribers' experience and knowledge, peer influence, pharmaceutical detailing, staff composition at organizations, and scientific literature influence uptake of new medicines [8-10]. However, the present review also highlighted that studies reporting factors affecting new medicine use lacked exploration of wider prescriber factors (e.g., motivation, values and goals, or beliefs about new medicines) and organizational factors (e.g., readiness for innovation, culture and climate, implementation process) reported in the implementation literature affecting implementation of health innovations [7, 87, 91]. Deficiency in reporting these factors could be due to the data sources used by the reviewed studies (mostly secondary administrative data from various databases) and a lack of theoretical frameworks used to inform study designs of reviewed studies. Only 20 of the reviewed studies referenced theoretical approaches employed but none of the studies addressed all constructs of the theoretical approach referenced. Future studies employing determinant frameworks or implementation theories [91] for primary data collection are required to address gaps in understanding barriers and facilitators to the implementation of new medicines into clinical practice.

\section{Strengths and limitations}

This systematic review had a broad search strategy over seven databases and included studies of all methodological designs, conducted in both primary and secondary care settings. Grey literature and non-English language articles were excluded for pragmatic reasons and only studies published from 2008 and onwards were included, so other relevant studies might have been missed. The synthesis was underpinned by a determinant framework used in implementation science, which allowed the conceptualisation of the findings as provided in the review. Most of the reviewed studies were medium (38 studies) or high (26 studies) quality increasing confidence in the review findings. Finally, included studies covered medicines with varied complexities and expertise required to prescribe them. Therefore, not all influential factors identified in the review are relevant to all healthcare settings and medicines, reducing the generalisability of the review findings. 


\section{Conclusions}

This systematic review provides a comprehensive exploration of factors affecting the use of new medicines and identified potential gaps in the research literature, through the use of a determinant framework used in implementation science. Factors affecting new medicine use not reported in earlier reviews were identified and included the following: patient influence and education level, cost of new medicines, formulary and reimbursement restrictions, and guidelines. Further research employing determinant frameworks or implementation theories are needed to address identified gaps, especially regarding wider patient, prescriber, and organizational factors, in understanding barriers and facilitators to the uptake of new medicines into clinical practice.

\section{Abbreviations}

PRISMA: Preferred Reporting Items for Systematic Reviews and MetaAnalyses; QATSDD: Quality Assessment Tool for Studies with Diverse Designs; GP: General Practitioner

\section{Supplementary Information}

The online version contains supplementary material available at https://doi. org/10.1186/s12913-021-07196-4.

Additional File 1. PRISMA checklist.

Additional File 2. Medline Search Strategy.

Additional File 3. Methodological quality of included studies using the QATSDD tool.

Additional File 4. Summary of thematic areas identified in the included studies.

\section{Acknowledgments}

Not applicable.

\section{Authors' contributions}

$K M, D P, S R$ and $K S$ contributed to the conception and design of the study. KM, DP, SR, and KS designed the literature search. KM performed the literature search. KM and JT conducted the screening and study selection process. KM, JT, and IM completed quality appraisal of included studies. KM, $I M, D P, S R$, and KS were involved in the data interpretation. KM wrote the first draft of the manuscript. DP, SR, KS, JT, and IM provided critical input and aided in the revision of the manuscript. All authors have read and approved the final manuscript.

\section{Funding}

This work presents research funded by the Pharmacy Research UK (grant reference: PRUK-2018-GA-1-KM) and Leeds Teaching Hospitals NHS Trust. The views expressed are those of the author and not necessarily those of Pharmacy Research UK or Leeds Teaching Hospitals NHS Trust. The funding source had no role in the design of the study, the data collection, the analysis, the interpretation of data or writing the manuscript.

\section{Availability of data and materials}

All data generated or analysed during this study are included in this published article and additional files including articles included in the analysis which are cited in the reference list.

\section{Declarations}

Ethics approval and consent to participate Not applicable.
Consent for publication

Not applicable.

\section{Competing interests}

The authors declare they have no competing interests.

\section{Author details}

${ }^{1}$ Medicine Optimisation Research Group, School of Pharmacy and Medical Sciences, University of Bradford, Richmond Road, Bradford BD7 1DP, UK. ${ }^{2}$ Medicine Management and Pharmacy Services, Leeds Teaching Hospitals NHS Trust, Leeds, UK. ${ }^{3}$ Department of Management, Huddersfield Business School, University of Huddersfield, Huddersfield HD1 3DH, UK.

Received: 30 March 2021 Accepted: 19 October 2021

Published online: 05 November 2021

\section{References}

1. Morris ZS, Wooding S, Grant J. The answer is 17 years, what is the question: understanding time lags in translational research. J Roy Soc Med. 2011; 104(12):510-20. https://doi.org/10.1258/jrsm.2011.110180.

2. Ewbank L, Omojomolo D, Sullivan K, McKenna $H$. The rising cost of medicines to the NHS: what is the story? The King's fund. 2018. https:// www.kingsfund.org.uk/sites/default/files/2018-04/Rising-cost-of-medicines. pdf?platform=hootsuite.

3. World Health Organization. WHO Health Innovation Group. https://www. who.int/life-course/about/who-health-innovation-group/en/. Accessed 28 May 2020.

4. Office for Life Sciences and Welcome Trust. Accelerated Access Review: Final Report. 2016. https://wellcome.ac.uk/what-we-do/our-work/accelera ted-access-review. Accessed 10 March 2017.

5. Office for Life Sciences. Life Sciences Competitiveness Indicators. 2019. https://assets.publishing.service.gov.uk/government/uploads/system/uploa ds/attachment_data/file/811347/life-sciences-competitiveness-data-2019.pdf. Accessed 29 May 2020.

6. Nilsen P. Making sense of implementation theories, models and frameworks Implement Sci. 2015;10(1):53. https://doi.org/10.1186/s13012-015-0242-0.

7. Chaudoir SR, Dugan AG, Barr CHI. Measuring factors affecting implementation of health innovations: a systematic review of structural, organizational, provider, patient, and innovation level measures. Implement Sci. 2013;8(1):22-42. https://doi.org/10.1186/1748-5908-8-22

8. Mason A. New medicines in primary care: a review of influences on general practitioner prescribing. J Clin Pharm Ther. 2008;3(1):1-10. https://doi.org/1 0.1111/j.1365-2710.2008.00875.x.

9. Chauhan D, Mason A. Factors affecting the uptake of new medicines in secondary care- a literature review. J Clin Pharm Ther. 2008;33(4):339-48. https://doi.org/10.1111/j.1365-2710.2008.00925.x.

10. Lubloy A. Factors affecting the uptake of new medicines: a systematic literature review. BMC Health Serv Res. 2014;14(1):469-94. https://doi.org/1 $0.1186 / 1472-6963-14-469$

11. Loughlin M, Buetow S, Cournoyea M, Copeland SM, Chin-Yee B, Fulford KWM. Interactions between persons - knowledge, decision making, and the co-production of practice. J Eval Clin Pract. 2019;25(6):911-20. https://doi. org/10.1111/jep.13297

12. Department of Health. High Quality Care for All: NHS Next Stage Review Final Report. 2008. https://assets.publishing.service.gov.uk/government/ uploads/system/uploads/attachment_data/file/228836/7432.pdf. Accessed 28 May 2020.

13. Medlinskiene K, Tomlinson J, Marques I. A systematic review of barriers and enablers to the uptake of new medicines into practice at organization level. PROSPERO 2018 CRD42018108536. https://www.crd.york.ac.uk/prospero/ display_record.php?ID=CRD42018108536.

14. Moher D, Liberati A, Tetzlaff J, Altman DG. The PRISMA group. Preferred Reporting Items for Systematic Reviews and Meta-Analyses: The PRISMA Statement PLoS Medicine. 2009;6(7):e1000097. https://doi.org/10.1371/ journal.pmed.1000097.

15. Liberati A, Altman DG, Tetzlaff J, Mulrow C, Gotzsche PC, Loannidis JPA, et al. The PRISMA statement for reporting systematic reviews and metaanalyses of studies that evaluate health care interventions: explanation and elaboration. PLoS Med. 2009:6(7):e1000100. https://doi.org/10.1371/journal. pmed. 1000100 . 
16. World Health Organization. Health topics: Innovation. https://www.who.int/ topics/innovation/en/. Accessed 28 May 2020.

17. Shamseer $L$, Moher D, Clarke M, Ghersi D, Liberati A, Petticrew $M$, et al. the PRISMA-P Group. Preferred reporting items for systematic review and metaanalysis protocols (PRISMA-P) 2015: elaboration and explanation. BMJ. 2015; 354:i4086.

18. Sirriyeh $R$, Lawton R, Gardner P, Armitage G. Reviewing studies with diverse designs: the development and evaluation of a new tool. J Eval Clin Pract. 2012;18(4):746-52. https://doi.org/10.1111/j.1365-2753.2011.01662.x.

19. Carroll C, Booth A, Leaviss J, Rick J. "Best fit" framework synthesis: refining the method. BMC Med Res Methodol. 2013;13(1):37. https://doi.org/10.11 86/1471-2288-13-37.

20. Ritchie J, Spencer L. Qualitative data analysis for applied policy research. In: Bryman A, Burgess RG, editors. Analyzing qualitative data. Routledge; 1994. p.173-194, DOl: https://doi.org/10.4324/9780203413081_chapter_9.

21. Abraham AJ, Knudsen HK, Rothrauff TC, Roman PM. The adoption of alcohol pharmacotherapies in the clinical trials network: the influence of research network participation. J Subst Abus Treat. 2010;38(3):275-83. https://doi. org/10.1016/j.jsat.2010.01.003

22. AbuDagga A, Stephenson JJ, Fu AC, Kwong WJ, Tan H, Weintraub WS. Characteristics affecting oral anticoagulant therapy choice among patients with non-valvular atrial fibrillation: a retrospective claims analysis. BMC Health Serv Res. 2014;14(1):310. https://doi.org/10.1186/1472-6963-14-310.

23. Anderson TS, Huskamp HA, Epstein AJ, Barry CL, Men A, Berndt ER, et al. Antipsychotic prescribing: do conflict of interest policies make a difference? Med Care. 2015;53(4):338-45. https://doi.org/10.1097/MLR.0000000000000329.

24. Anderson TS, Lo-Ciganic WH, Gellad WF, Zhang R, Huskamp HA, Choudhry $\mathrm{NK}$, et al. Patterns and predictors of physician adoption of new cardiovascular drugs. Healthcare. 2018;6(1):33-40. https://doi.org/10.1016/j. hjdsi.2017.09.004.

25. Baik SH, Hernandez I, Zhang Y. Evaluating the initiation of novel Oral anticoagulants in Medicare beneficiaries. J Manag Care Spec Pharm. 2016; 22(3):281-92. https://doi.org/10.18553/jmcp.2016.22.3.281.

26. Boon P, Dejonghe P, Legros B, Sadzot B, Rijckevorsel K, Shmedding E. Impact of reimbursement restrictions on the choice of antiepileptic drugs: Belgian study on epilepsy treatment (BESET). Seizure. 2008;17(4):350-7. https://doi.org/10.1016/j.seizure.2007.11.005.

27. Bourke J, Roper S. In with the new: the determinants of prescribing innovation by general practitioners in Ireland. Eur J Health Econ. 2012;3(4): 393-407. https://doi.org/10.1007/s10198-011-0311-5.

28. Brais C, Larochelle J, Turgeon MH, Blais L, Farand P, Perreault S, et al. Predictors of direct oral anticoagulants utilization for thromboembolism prevention in atrial fibrillation. J Pharm Pharm Sci. 2017;20:8-14. https://doi. org/10.18433/J30W4F.

29. Burden AM, Tadrous M, Calzavara A, Cadarette SM. Uptake and characteristics of zoledronic acid and denosumab patients and physicians in Ontario, Canada: impact of drug formulary access. Osteoporos Int. 2015; 26(5):1525-33. https://doi.org/10.1007/s00198-014-3023-8.

30. Carracedo-Martinez E, Pia-Moraneira A, Figueiras A. Trends in celecoxib and etoricoxib prescribing following removal of prior authorization requirement in Spain. J Clin Pharm Ther. 2017;42(2):185-8. https://doi. org/10.1111/jcpt.12490.

31. Chamberlain C, Collin SM, Stephens P, Donovan J, Bahl A, Hollingworth W. Does the cancer drugs fund lead to faster uptake of cost-effective drugs? A time-trend analysis comparing England and Wales. Br J Cancer. 2014;111(9): 1693-702. https://doi.org/10.1038/bjc.2014.86.

32. Chintagunta PK, Jiang $R$, Jin GZ. Information, learning, and drug diffusion: the case of Cox-2 inhibitors. Quant Mark Econ. 2009;7(4):399-443. https:// doi.org/10.1007/s11129-009-9072-1.

33. Chressanthis GA, Khedkar P, Jain N, Poddar P, Seiders MG. Can access limits on sales representatives to physicians affect clinical prescription decisions? A study of recent events with diabetes and lipid drugs. J Clin Hypertens. 2012;14(7):435-46. https://doi.org/10.1111/j.1751-7176.2012.00651.x.

34. Conti RM, Bernstein A, Meltzer DO. How do initial signals of quality influence the diffusion of new medical products? The case of new cancer treatments. Adv Health Econ Health Serv Res. 2012;23:123-48. https://doi. org/10.1108/50731-2199(2012)0000023008

35. DeVore AD, Hill CL, Thomas L, Sharma PP, Albert NM, Butler J, et al. Patient, provider, and practice characteristics associated with Sacubitril/valsartan use in the United States. Circ Heart Fail. 2018;11(9):e005400. https://doi.org/10.11 61/CIRCHEARTFAILURE. 118.005400.
36. Donohue JM, Guclu H, Gellad WF, Chang C-CH, Huskamp HA, Choudhry NK, et al. Influence of peer networks on physician adoption of new drugs. PLoS One. 2018;13(10):e0204826. https://doi.org/10.1371/journal.pone.0204826.

37. Ducharme $L$, Abraham AJ. State policy influence on the early diffusion of buprenorphine in community treatment programs. Subst Abuse Treat Prev Policy. 2008;3(1):17-27. https://doi.org/10.1186/1747-597X-3-17.

38. Dybdhal T, Sondergaard J, Kragstrup J, Kristiansen IS, Andersen M. Primary care physicians' adoption of new drugs is not associated with their clinical interests: a pharmacoepidemiologic study. Scand J Prim Health Care. 2011; 29(2):117-21. https://doi.org/10.3109/02813432.2011.570024.

39. Friedman PD, Jiang $L$, Alexander JA. Top manager effects on buprenorphine adoption in outpatient substance abuse treatment programs. J Behav Health Serv Res. 2010;37(3):322-37. https://doi.org/10.1007/s11414-009-9169z.

40. Fuksa $L$, Vocelka $M$, Vytrisalova $M$. The impact of changes in national prescribing conditions for statins on their public expenditure and utilization in the Czech Republic 1997-2013. Health Policy. 205(119):1255-64.

41. Garjon FJ, Azparren A, Vergara I, Azaola B, Loyassa JR. Adoption of new drugs by physicians: a survival analysis. BMC Health Serv Res. 2012;12(1):5666. https://doi.org/10.1186/1472-6963-12-56.

42. Groves KEM, Schellinck T, Sketris I, MacKinnon NJ. Identifying early prescribers of Cycloxygenase-2 inhibitors (COX-2s) in Nova Scotia, Canada: considerations for targeted academic detailing. Res Social Adm Pharm. 2010;6(3):257-67. https://doi.org/10.1016/j.sapharm.2009.09.003.

43. Haider SI, Johnell K, Weitoft G, Thorslund M, Fastbom J. Patient educational level and use of newly marketed drugs: a register-based study of over 600,000 older people. Eur J Clin Pharmacol. 2008;64(12):1215-22. https://doi. org/10.1007/s00228-008-0549-8.

44. Hickson RP, Cole AL, Dusetzina SB. Implications of removing Rosiglitazone's black box warning and restricted access program on the uptake of Thiazolidinediones and Dipeptidyl Peptidase-4 inhibitors among patients with type 2 diabetes. J Manag Care Spec Pharm. 2019;25(1):72-9. https://doi. org/10.18553/jmcp.2019.25.1.072.

45. Hirunrassamee S, Ratanawijitrasin S. Does your health care depend on how your insurer pays providers? Variation in utilization and outcomes in Thailand. Int J Health Care Finance Econ. 2009;9(2):153-68. https://doi.org/1 0.1007/s10754-009-9062-6.

46. Hsieh CR, Liu YM. Availability, healthcare costs, and utilization patterns of biologics in Taiwan. Value Health. 2012;15(Suppl 1):35-42. https://doi.org/1 0.1016/j.jval.2011.11.025

47. Huang C, Siu M, Vu L, Wong S, Shin J. Factors influencing doctors' selection of dabigatran in non-valvular atrial fibrillation. J Eval Clin Pract. 2013;19(5): 938-43. https://doi.org/10.1111/j.1365-2753.2012.01886.x.

48. Huskamp HA, O'Malley AJ, Horvitz-Lennon M, Taub AL, Berndt ER, Donohue $J M$. How quickly do physicians adopt new drugs? The case of secondgeneration antipsychotics. Psychiatr Serv. 2013;64(4):324-30. https://doi. org/10.1176/appi.ps.201200186.

49. Iyengar R, Van den Bulte C. Valente TW, Opinion leadership and social contagion in new product diffusion. Market Sci. 2011;30:195-212.

50. Karampli E, Souliotis K, Polyzos N, Chatzaki E. Why do physicians prescribe new antidiabetic drugs? A qualitative study in the Greek healthcare setting. Health Policy and Technology. 2020;9(2):166-73. https://doi.org/10.1016/j. hlpt.2020.02.007.

51. Keating NL, Huskamp HA, Schrag D, McWilliams JM, McNeil BJ, Landon BE, et al. Diffusion of Bevacizumab across oncology practices: an observational study. Med Care. 2018;56(1):69-77. https://doi.org/10.1097/MLR. 0000000000000840

52. Keating NL, O'Malley AJ, Onnela J-P, Gray SW, Bruce E. Association of physician peer influence with subsequent physician adoption and use of bevacizumab. JAMA Netw Open. 2020;3(1):e1918586. https://doi.org/10.1 001/jamanetworkopen.2019.18586.

53. Kennedy C, Choitir CN, Clarke S, Bennett K, Barry M. Direct oral anticoagulants uptake and an oral anticoagulation paradox. Br J Clin Pharmacol. 2020;86(2):392-7. https://doi.org/10.1111/bcp.14171.

54. Kereszturi JL, Lubloy A, Benedek G. Examination of pharmaceutical innovation diffusion using the cox model. Hungarian Statistical Review. 2015;93:47-66.

55. King M, Essick C, Bearman P, Cole J, Ross JS. Medical school gift restriction policies and physician prescribing of newly marketed psychotropic medications: difference-in-differences analysis. BMJ. 2013:346(jan30 5):f264. https://doi.org/10.1136/bmj.f264. 
56. King M, Bearman PS. Gifts and influence: conflict of interest policies and prescribing of psychotropic medications in the United States. Soc Sci Med. 2017;172:153-62. https://doi.org/10.1016/j.socscimed.2016.11.010.

57. Knudsen HK, Abraham AJ, Johnson JA, Roman PM. Buprenorphine adoption in the National Drug Abuse Treatment Clinical Trials Network. J Subst Abus Treat. 2009;37(3):307-12. https://doi.org/10.1016/j.jsat.2008.12.004.

58. Lin HC, Erickson SR, Balkrishnan R. Physician prescribing patterns of innovative antidepressants in the United States: the case of MDD patients 1993-2007. Int J Psychiatry Med. 2011;42(4):353-68. https://doi.org/10.2190/ PM.42.4.b.

59. Lin SJ, Jan KA, Kao JT. Colleague interactions and new drug prescribing behavior: the case of the initial prescription of antidepressants in Taiwanese medical centers. Soc Sci Med. 2011;73(8):1208-13. https://doi.org/10.1016/j. socscimed.2011.06.065

60. Liu YM, Yang YHK, Hsieh CR. The determinants of the adoption of pharmaceutical innovation: evidence from Taiwan. Soc Sci Med. 2011;72(6): 919-27. https://doi.org/10.1016/j.socscimed.2010.12.027.

61. Liu Q, Gupta S. A micro-level diffusion model for new drug adoption. J Prod Innovat Manag. 2012;29(3):372-84. https://doi.org/10.1111/j.1540-5885.2012. 00912.x.

62. Lo-Ciganic WH, Gellad WF, Huskamp HA, Choudhry NK, Chang CCH, Zhang $\mathrm{R}$, et al. Who were the early adopters of dabigatran? An application of group-based trajectory models. Med Care. 2016;54(7):725-32. https://doi org/10.1097/MLR.0000000000000549.

63. Luo N, Fonarow GC, Lippmann SJ, Mi X, Heidenreich PA, Yancy CW, et al. Early adoption of Sacubitril/valsartan for patients with heart failure with reduced ejection fraction: insights from get with the guidelines-heart failure (GWTG-HF). JACC Heart Fail. 2017;5(4):305-9. https://doi.org/10.1016/j.jchf.2 016.12.018.

64. Luo N, Ballew NG, O'Brien EC, Greiner MA, Peterson PN, Hammill BG, et al. Early impact of guideline publication on angiotensin-receptor neprilysin inhibitor use among patients hospitalized for heart failure. Am Heart J. 2018; 200:134-40. https://doi.org/10.1016/j.ahj.2018.01.009.

65. Luo N, Lippmann SJ, Mentz RJ, Greiner MA, Hammill BG, Hardy NC, et al. Relationship between hospital characteristics and early adoption of angiotensin-receptor/neprilysin inhibitor among eligible patients hospitalized for heart failure. J Am Heart Assoc. 2019;8(3):e010484. https:// doi.org/10.1161/JAHA.118.010484.

66. Manchanda $\mathrm{P}$, Xie $\mathrm{Y}$, Youn N. The role of targeted communication and contagion in product adoption. INFORMS. 2008;27(6):961-76. https://doi. org/10.1287/mksc.1070.0354

67. Martin E, Pourtau L, Di Palma M, Delaloge S. New oral targeted therapies for metastatic breast cancer disrupt the traditional patients' management-a healthcare providers' view. Eur J Cancer Care. 2017;26(6):e12624-33. https:// doi.org/10.1111/ecc.12624.

68. Murphy A, Kirby A, Bradley C. Knowledge is power: general practitioners prescribing of new oral anticoagulants in Ireland. BMC Res Notes. 2018; 11(1):478-84. https://doi.org/10.1186/s13104-018-3597-x.

69. Netherland J, Botsko M, Egan JE, Saxon AJ, Cunnigham CO, Finkelstein R, et al. The BHIVES collaborative. Factors affecting willingness to provide buprenorphine treatment. J Subst Abus Treat. 2009;36(3):244-51. https://doi. org/10.1016/j.jsat.2008.06.006.

70. Ohl M, Lund B, Belperio PS, Goetz MB, Rimland D, Richardson K, et al. Rural residence and adoption of a novel HIV therapy in a national, equal-access healthcare system. AIDS Behav. 2013;17(1):250-9. https://doi.org/10.1007/s1 0461-011-0107-8.

71. Ohlsson H, Chaix B, Merlo J. Therapeutic traditions, patient socioeconomic characteristics and physicians' early new drug prescribing-a multilevel analysis of rosuvastatin prescription in South Sweden. Eur J Clin Pharmacol. 2009;65(2):141-50. https://doi.org/10.1007/s00228-008-0569-4.

72. Patel PA, Zhao X, Fonarow GC, Lytle BL, Smith EE, Xian Y, et al. Novel oral anticoagulant use among patients with atrial fibrillation hospitalized with ischemic stroke or transient ischemic attack. Circ-Cardiovas Qual. 2015;8(4): 383-92. https://doi.org/10.1161/CIRCOUTCOMES.114.000907.

73. Potpara TS, Trendafilova E, Dan GA, Goda a, Kusljugic Z, Manola S, et al. lip GYH, BALKAN-AF investigators. The patterns of non-vitamin K antagonist Oral anticoagulants (NOACs) use in patients with atrial fibrillation in seven Balkan countries: a report from the BALKAN-AF survey. Adv Ther. 2017;34(8): 2043-57. https://doi.org/10.1007/s12325-017-0589-5.

74. Rodwin BA, Lu D, Giaimo A, Annapureddy A, Daggubati R, Curtis J, et al. Patient and hospital characteristics associated with ticagrelor uptake in acute MI: an analysis of the chest pain-MI registry. Int J Cardiol. 2020;304:1420. https://doi.org/10.1016/j.jjcard.2020.01.029.

75. Sato D, Sato Y, Masuda S, Kimura H. Impact of the sitagliptin alert on prescription of oral antihyperglycemic drugs in Japan. Int J Clin Pharm. 2012;34(6):917-24. https://doi.org/10.1007/s11096-012-9694-3.

76. Savage SA, Abraham AJ, Knudsen HK, Rothrauff TC, Roman PM. Timing of buprenorphine adoption by privately funded substance abuse treatment programs: the role of institutional and resource-based interorganizational linkages. J Subst Abus Treat. 2012;42(1):6-24. https://doi.org/10.1016/j.jsat.2 011.06.009.

77. Scholten N, Pfaff H, Lehmann HC, Fink GR, Karbach U. Who does it first? The uptake of medical innovations in the performance of thrombolysis on ischemic stroke patients in Germany: a study based on hospital quality data Implement Sci. 2015;10(1):10. https://doi.org/10.1186/s13012-014-0196-7.

78. Steinberg BA, Holmes DN, Piccini JP, Ansell J, Chang P, Fonarow GC, et al. Early adoption of dabigatran and its dosing in US patients with atrial fibrillation: results from the outcomes registry for better informed treatment of atrial fibrillation. J Am Heart Assoc. 2013;2(6):e000535-47. https://doi. org/10.1161/JAHA.113.000535.

79. Tanislav C, Allendorfer J, Pfeilschifter W, Fuest S, Stein M, Meyer M, et al. One decade of oral anticoagulation in stroke patients: results from a large country-wide hospital-based registry. Int J Stroke. 2018;13(3):308-12. https:// doi.org/10.1177/1747493017733928.

80. Tobin L, Neto AA, Wutzke S, Patterson C. Influences on the prescribing of new drugs. Aust Fam Physician. 2008;37:78-81.

81. Tsai YW, Wen YW, Huang WF, Kuo KN, Chen PF, Shih HW, et al. Pharmaceutical penetration of new drug and pharmaceutical market structure in Taiwan: hospital-level prescription of thiazolidinediones for diabetes. Eur J Health Econ. 2010;11(3):279-90. https://doi.org/10.1007/s101 98-009-0174-1.

82. Wang PJ, Chou YJ, Lee CH, Pu C. Diffusion of new medication across different income groups under a universal health insurance program: an example involving newly enlisted nonsteroidal anti-inflammatory drugs for elderly osteoarthritis patients. Int J Public Health. 2010;55(5):497-506. https://doi.org/10.1007/s00038-010-0132-9.

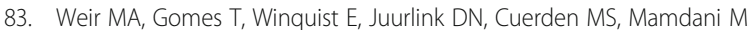
Effects of funding policy changes and health warnings on the use of erythropoiesis-stimulating agents. J Oncol Pract. 2012;8(3):179-84. https:// doi.org/10.1200/JOP.2011.000287.

84. Wen YW, Huang WF, Lee YC, Kuo KN, Tsai CR, Tsai YW. Diffusion patterns of new antidiabetic drugs into hospitals in Taiwan: the case of Thiazolidinediones for diabetes. BMC Health Serv Res. 2011;11(1):21-34. https://doi.org/10.1186/1472-6963-11-21.

85. Zhang Y, Méndez SJ, Scott A. Factors affecting general practitioners' decisions to adopt new prescription drugs - cohort analyses using Australian longitudinal physician survey data. BMC Health Serv Res. 2019; 19(1):94. https://doi.org/10.1186/s12913-019-3889-4.

86. Zhang B, Song Y, Fu Y, Zhu B, Wang B, Wang J. Current status of the clinical use of PD-1/PD-L1 inhibitors: a questionnaire survey of oncologists in China. BMC Cancer. 2020;20(1):86. https://doi.org/10.1186/ s12885-020-6583-3.

87. Damschroder LJ, Aron DC, Keith RE, Kirsh SR, Alexander JA, Lowery JC. Fostering implementation of health services research findings into practice: a consolidated framework for advancing implementation science. Implement Sci. 2009;4(1):50. https://doi.org/10.1186/1748-5908-4-50.

88. Jaakkola E, Renko M. Critical innovation characteristics influencing the acceptability of a new pharmaceutical product format. J Mark Manag. 2007; 23(3-4):327-46. https://doi.org/10.1362/026725707X196404.

89. Hahn RA, Truman Bl. Education improves public health and promotes health equity. Int J Health Serv. 2015;45(4):657-78. https://doi.org/10.1177/ 0020731415585986.

90. Godman B, Bucsics A, Vella Bonanno P, Oortwijn W, Rothe CC, Ferrario A, Bosselli S, Hill A, Martin AP, Simoens S, Kurdi A, Gad M, Gulbinovič J, Timoney A, Bochenek T, Salem A, Hoxha I, Sauermann R, Massele A, Guerra AA Jr, Petrova G, Mitkova Z, Achniotou G, Laius O, Sermet C, Selke G, Kourafalos V, Yfantopoulos J, Magnusson E, Joppi R, Oluka M, Kwon H-Y, Jakupi A, Kalemeera F, Fadare JO, Melien O, Pomorski M, Wladysiuk M, Marković-Peković V, Mardare I, Meshkov D, Novakovic T, Fürst J, Tomek D, Zara C, Diogene E, Meyer JC, Malmström RE, Wettermark B, Matsebula Z, Campbell S and Haycox A. Barriers for Access to New Medicines: Searching for the Balance Between Rising Costs and Limited Budgets. Front. Public 
Health 2018;6:328. doi: https://doi.org/10.3389/fpubh.2018.00328. Accessed 22 December 2020.

91. Nilsen P. Making sense of implementation theories, models and frameworks. Implement Sci. 2015;10(1):53. https://doi.org/10.1186/s13012-015-0242-0.

\section{Publisher's Note}

Springer Nature remains neutral with regard to jurisdictional claims in published maps and institutional affiliations.

Ready to submit your research? Choose BMC and benefit from:

- fast, convenient online submission

- thorough peer review by experienced researchers in your field

- rapid publication on acceptance

- support for research data, including large and complex data types

- gold Open Access which fosters wider collaboration and increased citations

- maximum visibility for your research: over $100 \mathrm{M}$ website views per year

At $B M C$, research is always in progress.

Learn more biomedcentral.com/submissions 